\title{
Sincronía de tiempos rituales y solares en las estructuras de 4 x 65 días de los códices mayas
}

\section{Synchrony of Ritual and Solar Times in the 4 x 65-Day Structures of the Maya Codices}

\author{
Carlos Barrera Atuesta \\ Investigador independiente
}

\begin{abstract}
Resumen: Este documento presenta una metodología viable para mantener en sincronía fechas solares características del ciclo agrícola mesoamericano con reconocidos ciclos calendáricos y celestes. El procedimiento establecido combina técnicas simples de aritmética modular con el desarrollo de estructuras de 4 x 65 días centradas sobre la referencia solar de origen más probable del calendario civil maya: el solsticio de invierno. Los resultados obtenidos revelan nuevas dimensiones de cómputo entre fechas rituales, representadas por múltiples intervalos canónicos y astronómicos que se desarrollan paralelamente en ambos sentidos del tiempo. El alcance general de los intervalos oscila entre el orden de los días y los siglos, y su precisión suele ser menor de \pm 1 día.
\end{abstract}

Palabras clave: cuenta ritual de 260 días, astronomía maya, ciclos agrícolas, códices mayas, calendarios de horizonte.

\begin{abstract}
This paper presents a viable methodology to maintain synchronicity between solar dates characteristic of the Mesoamerican agricultural cycle with recognized calendrical and celestial cycles. The established procedure combines simple techniques of modular arithmetic with the development of 4 x 65 day-structures centered on the solar reference of most probable origin of the Maya civil calendar: the winter solstice. The results obtained reveal new dimensions of computation between ritual dates, represented by multiple canonical and astronomical intervals that develop in parallel in both directions of time. The general scope of the intervals ranges from the order of days to centuries, and their accuracy is usually less than \pm 1 day.
\end{abstract}

Keywords: 260-day ritual count, Maya astronomy, agricultural cycles, Maya codices, horizon-based calendars.

RECEPCIÓN: 6 de diciembre de 2017.

ACEPTACIÓN: 17 de abril de 2018.

DOI: 10.19130/iifl.ecm.2019.53.951. 


\section{Introducción}

Los códices mayas de Dresde y Madrid registran almanaques y tablas de $4 \mathrm{x}$ 65 días (Aveni, Morandi y Peterson, 1995: tablas 1a y 1b; Vail y Aveni, 2004: tabla 8.1, 221s; Bricker y Bricker, 2011: 660-674), cuya iconografía y estructura concuerdan con rituales agrícolas de agua y fuego descritos en textos coloniales (Rice, 2004: 245-248; Bricker, 2007: 37-39; Morales, 2014). Las principales fechas solares que definen dichos intervalos rituales, según lo informan distintos estudios etnográficos, coinciden con puntos del horizonte hacia los que se orientan numerosas estructuras arquitectónicas prehispánicas (Sánchez y Šprajc, 2015: 89-105; Vail y Looper, 2015; Méndez, 2012). De esta forma, las mismas fechas utilizadas en la Mesoamérica ancestral para delimitar las temporadas de lluvia y sequía, y para señalar los momentos propicios para la siembra, cosecha y recolección, habrían sido perpetuadas por medio de estas construcciones, o por otros elementos orientados hacia el cenit o el horizonte (Šprajc, 2017; Broda, 2016; Wood y Márquez, 2016; Galindo, 2015; Carter, 2014: 35-38; Aldana y Fash, 2014; Green, 2014; Aveni, 2013; Montero, 2013; Grofe, 2012; Zaro y Lohse, 2005; Aveni, Dowd y Vining, 2003; Espinosa, 2002; Morante, 2001; Soruco, 1991; Aveni y Hartung, 1986, 1976, 1975; Hohmann y Vogrin, 1982; Tichy, 1978; Drucker, 1977; Hartung, 1968; Morley, 1920).

Estas efectivas técnicas de seguimiento solar no satisfacen, sin embargo, una importante conclusión extractada por la doctora Johanna Broda $(1971,1983)$ luego de observar la "correspondencia entre los ritos, los fenómenos climatológicos y la agricultura” (Šprajc, 2000: 135): "debe haber existido algún método, aún desconocido, para mantener el calendario en concordancia con el año solar" (Broda, 1983: 145). (Contrástese la posición de Broda con otros estudios sobre años solares de Iwaniszewski, 2013; Šprajc, 2000; Bolles, 1990 y Teeple, 1931.)

La metodología aquí propuesta plantea una solución viable para los referidos problemas de sincronía, partiendo del análisis de estructuras de $4 \times 65^{\mathrm{d}}$ inscritas en códices prehispánicos y podría reflejar, por ende, algunos aspectos perdidos del conocimiento indígena atávico.

\section{Justificación}

La importancia de la sincronía entre fechas solares y ciclos calendáricos y astronómicos, se fundamenta principalmente en razones rituales y de subsistencia. Para los antiguos mayas (y demás culturas afines de Mesoamérica) resultaba conveniente establecer fechas canónicas de referencia coincidentes con fenómenos climatológicos periódicos que eran determinantes para los procesos agrícolas, como las temporadas de lluvia y sequía o los tiempos considerados propicios para sembrar y cosechar. Conscientes de que el calendario Jaab' y el año trópico carecían de concordancia permanente, y ante el riesgo que implicaba tener 
que regular sus actividades agrícolas esenciales dependiendo exclusivamente de observaciones astronómicas expuestas al albur de las condiciones atmosféricas, los antiguos habitantes de Mesoamérica habrían experimentado la necesidad de encontrar algún tipo de correspondencia solar a través de ciclos canónicos alternativos, como la cuenta ritual de $260^{\mathrm{d}}$, de hecho su opción más probable. $\mathrm{Al}$ respecto, Šprajc (2000: 151 s.) comenta y cita lo siguiente:

Asimismo parece significativo que en algunas comunidades indígenas que conservan las formas menos distorsionadas del calendario prehispánico, el año de 365 días tiene poca importancia para la regulación de los trabajos agrícolas; es el ciclo ritual de 260 días el que sirve para estos fines, en combinación con las observaciones astronómicas. ${ }^{1}$

El agricultor, por su parte, ha de procurar que la quema se lleve a cabo antes de que lleguen las primeras lluvias, pues, de lo contrario, quedaría imposibilitado para hacerlo, perdiendo así la ocasión de usar el terreno talado. Para preservarse de este peligro, el milpero suele acudir a alguno de los dos escribas que hay en el cacicazgo, el cual, usando un almanaque impreso en Mérida, le puede anunciar la clase de tiempo que ha de hacer en cada uno de los meses venideros.

De ello podría colegirse que dicha combinación de técnicas rituales-astronómicas acabó por producir un método eficaz de seguimiento del año trópico fundamentado en el uso de la cuenta ritual de $260^{\mathrm{d}}$, u otro "ciclo maya" útil. Las observaciones astronómicas, por su parte, habrían cumplido una importante función adicional de calibración, necesaria para validar y optimizar la pretendida sincronía entre ciclos. Sin perjuicio de lo anterior, en siglos posteriores a la ocupación española los agricultores se habrían visto obligados a conciliar sus rituales ancestrales con doctrinas católicas impuestas, ejerciendo un conveniente sincretismo a través del cual determinados "días de Santos" establecidos en el calendario cristiano habrían pasado a desempeñar las funciones inherentes a su ciclo ritual (Méndez, 2012; Sánchez y Šprajc, 2015: 94-97, 100s).

Conforme a los resultados obtenidos durante esta investigación, se infiere asimismo que la metodología aquí propuesta podría ser un instrumento útil para articular diversos modelos de representación e interpretación solar basados en el estudio de orientaciones astronómicas, cámaras cenitales y calendarios observacionales.

\section{Fundamentos y métodos}

Los métodos aquí desarrollados se inspiran en ciertos almanaques contenidos en los códices mayas de Dresde y Madrid que dividen la duración del ciclo ritual de $260^{\mathrm{d}}$ en cuatro intervalos equivalentes de $65^{\mathrm{d}}$. Estas particiones pueden estar conformadas a su vez por trayectos o distancias menores, técnicamente denominadas "Números de Distancia".

${ }^{1}$ Cursivas en el original. 
Los almanaques de $4 \times 65^{\mathrm{d}}$

La Figura 1 ilustra un almanaque típico de $4 \times 65^{\mathrm{d}}$ que se caracteriza por representar en cada una de sus particiones los cuatro rumbos y colores principales de la cosmovisión maya: rojo al oriente, blanco al norte, negro al occidente y amarillo al sur. Los respectivos textos jeroglíficos del almanaque, inscritos a doble columna, confirman dicha información y asocian estas cuatro direcciones cosmológicas con distintas manifestaciones de Chaak, el dios de la lluvia.

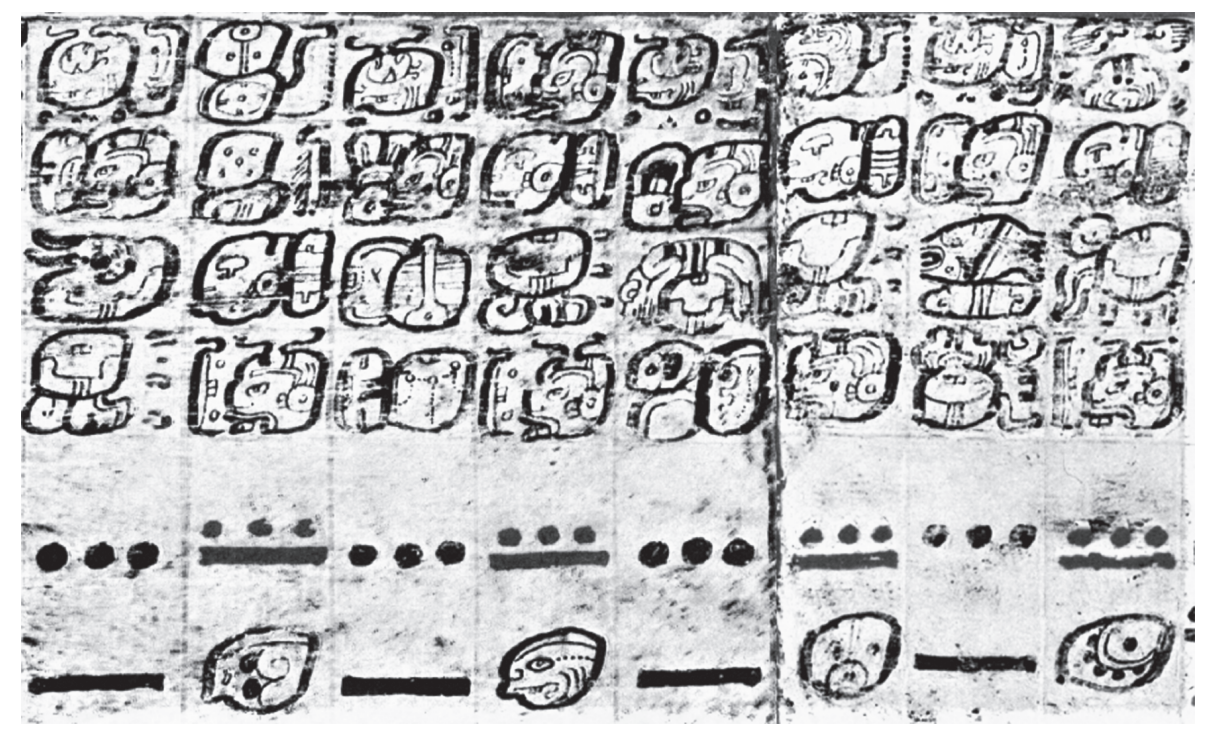

Figura 1. Almanaque D.30b-31b (Códice de Dresde).

La estructura de este almanaque —delineado en el sector central de las páginas 30 y 31 del Códice de Dresde - se ilustra en la Tabla 1, en donde sendos intervalos de 65d separan las fechas 8 Chikchan, 8 Ok, 8 Men y 8 Ajaw del ciclo ritual. El Número de Distancia de 65d que, desde 8 Ajaw conduce hacia 8 Chikchan, representa el completamiento de un ciclo ritual de $260 \mathrm{~d}$, a partir del cual, la secuencia descrita por la estructura del almanaque D.30b-31b podría repetirse ad infinitum. Para fines de diferenciación, las sucesivas fechas 8 Chikchan, así obtenidas, podrían registrase en renglones consecutivos, bajo una misma columna.

Una estructura de estas características estaría conformada, en consecuencia, por intervalos horizontales de $65^{\mathrm{d}}$ e intervalos verticales de $260^{\mathrm{d}}$, y se le podría denominar "estructura de $65^{\mathrm{d}}$ x $260^{\mathrm{d}}$ ". Sobre un sistema reticular de tales características se fundamenta este proyecto. 


\begin{tabular}{|c|c|c|c|c|c|c|c|}
\hline \multicolumn{2}{|c|}{ A } & \multicolumn{2}{c|}{ B } & \multicolumn{2}{c|}{ C } & \multicolumn{2}{c|}{ D } \\
\hline $\begin{array}{c}8 \\
\text { Chikchan }\end{array}$ & +65 & $\begin{array}{c}8 \\
\text { Ok }\end{array}$ & +65 & $\begin{array}{c}8 \\
\text { Men }\end{array}$ & +65 & $\begin{array}{c}8 \\
\text { Ajaw }\end{array}$ & +65 \\
\hline+260 & +260 & & +260 & & +260 & \\
\hline $\begin{array}{c}8 \\
\text { Chikchan }\end{array}$ & +65 & $\begin{array}{c}8 \\
\text { Ok }\end{array}$ & +65 & $\begin{array}{c}8 \\
\text { Men }\end{array}$ & +65 & $\begin{array}{c}8 \\
\text { Ajaw }\end{array}$ & +65 \\
\hline $\begin{array}{c}860 \\
8\end{array}$ & +260 & & +260 & & +260 & \\
\hline Chikchan & +65 & $\begin{array}{c}8 \\
\text { Ok }\end{array}$ & +65 & $\begin{array}{c}8 \\
\text { Men }\end{array}$ & +65 & $\begin{array}{c}8 \\
\text { Ajaw }\end{array}$ & +65 \\
\hline
\end{tabular}

Tabla 1. Estructura de $\left(4 \times 65^{\mathrm{d}}\right) \times 260^{\mathrm{d}}$ del almanaque D.30b-31b. Autor: Carlos Barrera Atuesta.

El módulo aritmético

El módulo aritmético es una herramienta simple, pero poderosa, para el análisis de fenómenos cíclicos. Por definición, el módulo $A$ de un número $B$ es el residuo $r$ que se obtiene al dividir $B$ entre $A$, y su expresión matemática es $B \bmod A=r$

Una aplicación práctica de este operador aritmético podría ser la siguiente: supóngase que es domingo y se desea saber qué día de la semana será dentro de $40^{\mathrm{d}}$. Como la semana representa un período de $7^{\mathrm{d}}$, entonces todo múltiplo entero de $7^{\mathrm{d}}$ conducirá de nuevo hacia un día domingo. Por tal motivo, después de transcurridos $5 \times 7^{\mathrm{d}}=35^{\mathrm{d}}$, aún será domingo, y se requerirán $5^{\mathrm{d}}$ adicionales para completar los $40^{\mathrm{d}}$ pretendidos. Esto se traduce, por supuesto, en que $40^{\mathrm{d}}$ después de un día domingo se alcanzará un día viernes. Habría bastado, por tanto, con avanzar únicamente $5^{\mathrm{d}}$ desde el domingo de origen para conocer la respuesta; dato que hubiésemos obtenido directamente de haber efectuado la operación $40 \bmod 7=5$.

Obsérvese ahora qué hubiese sucedido una semana después de aquel domingo situado a $5 \times 7^{\mathrm{d}}$ del origen. Los días transcurridos habrían sido $6 \times 7^{\mathrm{d}}=42^{\mathrm{d}}$, y no los $35^{\mathrm{d}}$ inicialmente considerados, así que los $40^{\mathrm{d}}$ en cuestión se habrían celebrado tan solo un par de días antes del cumplimiento de la sexta semana. Con base en el anterior análisis se podría definir entonces que, en un contexto cíclico, existen dos direcciones válidas de solución, una positiva y otra negativa. La solución negativa (-2) se obtiene al sustraer el valor del módulo (7) de la solución positiva (5).

De forma un poco más técnica, el uso del operador módulo (mod) se podría describir en función de los siguientes elementos relacionados con la división de 
los números $B / A$ : Dividendo $(B)$, divisor $(A)$, cociente $(C)$ y residuo $(r)$, por lo que la relación operacional se cumple del siguiente modo: $B=(C \times A)+r$.

Para una secuencia cíclica y de modo genérico, el cociente $C$ indica el número de ciclos completos, y el residuo $r$, el avance del número $B$ dentro del ciclo siguiente. Por tanto, para poder conocer qué cantidad $R$ falta para completar el ciclo siguiente, se requiere calcular $R=A-r$. Resulta más práctico, sin embargo, reformular dicha expresión en función de una cantidad erre prima $\left(R^{\prime}\right)$ de días, correspondiente a la ecuación $R^{\prime}=r-A$, para que el signo negativo del resultado indique que se trata de una posición anterior. Tenemos entonces que el módulo aritmético indica el orden dentro de algún ciclo, por lo que si $B=40$ y $A=7$ (datos del ejemplo inicial), se tendrá entonces que el residuo $r=5$ indica el quinto día (viernes) después del día 0 (domingo).

Ahora bien, sabemos por inscripciones epigráficas que los mayas del período Clásico equiparaban la fecha del "fin de período" anterior con el "asiento" u origen del período en curso (ver Figura 2, donde la inscripción cronológica de Palenque 13.0.1.9.2 $13 \mathrm{I} \mathrm{k}^{\prime}$ homologaba la fecha $20 \mathrm{Mol}$ del ciclo anterior con el origen 0 Ch'en del ciclo en curso).

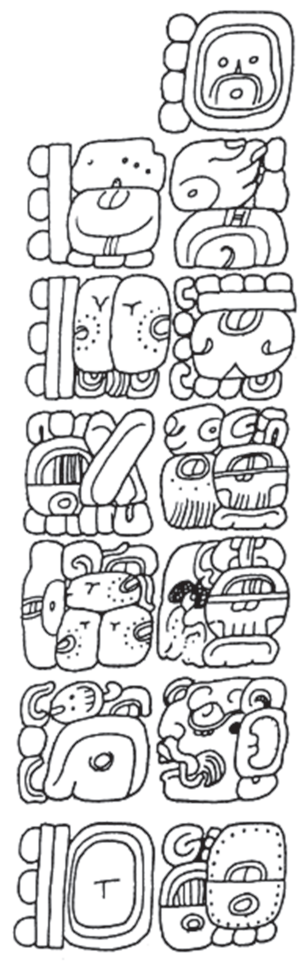

Figura 2. Registro cronológico 13.0.1.9.2 $13 \mathrm{I}$ ' 0 Ch'en (asiento de Ch'en) inscrito en el panel del Templo de la Cruz de Palenque como 13.0.1.9.2 13 lk' 20 Mol (fin de $\mathrm{Mol}$ )

(Según Linda Schele). 
Al aplicar este mismo criterio al día domingo de nuestro ejercicio, tenemos entonces que su posición puede ser representada como cero, pero también como siete. Es por esta razón que justificábamos la existencia de una "solución negativa” (más corta) que podríamos definir en función de una cantidad $R$ ' (erre prima) de días, expresable mediante la ecuación $R^{\prime}=r-A$, cuya operación resultante $(-2)$ indica la posición del día quinto (viernes) respecto del día séptimo (domingo).

En las Tablas 2 y 3 se relacionan los principales intervalos utilizados en el artículo, y la forma en que éstos se ven afectados por el operador aritmético "módulo" (mod), detallando para cada caso los elementos involucrados en el proceso. Los valores positivos de los intervalos se registran en la Tabla 2, y los valores negativos, en la Tabla 3. Nótese el intercambio de valores entre residuos $(r)$ y complementos $(R)$, así como la diferencia en el número de ciclos completos cuando un intervalo pasa de ser positivo a negativo (siendo el cociente $C$ la función entero de $B / A$ ).

\begin{tabular}{|c|c|c|c|c|c|c|c|}
\hline$n$ & $K$ & $B=n K$ & $A$ & $C$ & $r$ & $R$ & $R^{\prime}$ \\
\hline 2 & 20 & 40 & 7 & 5 & 5 & 2 & -2 \\
\hline 56 & 65 & 3640 & 365.242253 & 9 & 352.819723 & 12.422530 & -12.422530 \\
\hline 59 & 65 & 3835 & 365.242275 & 10 & 182.577250 & 182.665025 & -182.665025 \\
\hline 112 & 65 & 7280 & 365.242253 & 19 & 340.397193 & 24.845060 & -24.845060 \\
\hline 118 & 65 & 7670 & 365.242275 & 20 & 365.154500 & 0.087775 & -0.087775 \\
\hline 236 & 65 & 15340 & 365.242275 & 41 & 365.066725 & 0.175550 & -0.175550 \\
\hline 292 & 65 & 18980 & 365.242253 & 51 & 352.645097 & 12.597156 & -12.597156 \\
\hline 360 & 65 & 23400 & 365.242253 & 64 & 24.495808 & 340.746445 & -340.746445 \\
\hline 472 & 65 & 30680 & 365.242253 & 83 & 364.893001 & 0.349252 & -0.349252 \\
\hline 584 & 65 & 37960 & 365.242253 & 103 & 340.047941 & 25.194312 & -25.194312 \\
\hline
\end{tabular}

Tabla 2. Relación y análisis de los principales intervalos utilizados en el artículo, y detalle de los valores obtenidos para cada uno de los elementos que conforman el operador módulo.

Autor: Carlos Barrera Atuesta. 


\begin{tabular}{|c|c|c|c|c|c|c|c|}
\hline $\mathbf{n}$ & $\mathrm{K}$ & $\mathbf{B}=\mathrm{nK}$ & $\mathbf{A}$ & $\mathrm{C}$ & $\mathbf{r}$ & $\mathbf{R}$ & $\mathbf{R}^{\prime}$ \\
\hline-2 & 20 & -40 & 7 & -6 & 2 & 5 & -5 \\
\hline-56 & 65 & -3640 & 365.242253 & -10 & 12.422530 & 352.819723 & -352.819723 \\
\hline-59 & 65 & -3835 & 365.242275 & -11 & 182.665025 & 182.577250 & -182.577250 \\
\hline-112 & 65 & -7280 & 365.242253 & -20 & 24.845060 & 340.397193 & -340.397193 \\
\hline-118 & 65 & -7670 & 365.242275 & -21 & 0.087775 & 365.154500 & -365.154500 \\
\hline-236 & 65 & -15340 & 365.242275 & -42 & 0.175550 & 365.066725 & -365.066725 \\
\hline-292 & 65 & -18980 & 365.242253 & -52 & 12.597156 & 352.645097 & -352.645097 \\
\hline-360 & 65 & -23400 & 365.242253 & -65 & 340.746445 & 24.495808 & -24.495808 \\
\hline-472 & 65 & -30680 & 365.242253 & -84 & 0.349252 & 364.893001 & -364.893001 \\
\hline-584 & 65 & -37960 & 365.242253 & -104 & 25.194312 & 340.047941 & -340.047941 \\
\hline
\end{tabular}

Tabla 3. Relación y análisis de los principales intervalos utilizados en el artículo, y detalle de los valores obtenidos para cada uno de los elementos implícitos del operador módulo.

El cociente $C$ (ciclos completos) ha sido obtenido al aplicar la función entero $(Z)$ a $(B / A)$. Autor: Carlos Barrera Atuesta.

En dichas tablas, el valor $n$ indica el número (y la dirección) de las repeticiones aplicadas al factor $K$, que aquí describe intervalos múltiplos de $13^{\mathrm{d}}$ y $20^{\mathrm{d}}$, pero que también podría ser utilizado para representar otros múltiplos reconocidos de $7^{\mathrm{d}}$ y $9^{\mathrm{d}}$.

\section{Desarrollo de estructuras de $65^{d} \times 260^{d}$}

A partir del solsticio de invierno de diciembre 21 -la referencia natural de origen más probable del calendario solar maya - (Bricker, 1982; Tichy, 1991; Sánchez y Šprajc, 2015: 89 s.), se adicionan y sustraen intervalos múltiplos de $65^{\mathrm{d}}$, procediendo a registrar las fechas solares obtenidas dentro de un sistema coordenado en donde cada unidad básica de desplazamiento representa $65^{\mathrm{d}}$ en la escala horizontal, y $260^{\mathrm{d}}$ en la escala vertical (Figura 3). Bajo este tipo de ordenamiento, el avance del tiempo se define siguiendo la direccionalidad de la lectura (de izquierda a derecha y de arriba hacia abajo). La distribución simétrica de celdas en torno al solsticio de referencia facilita la identificación de patrones de distancia. 


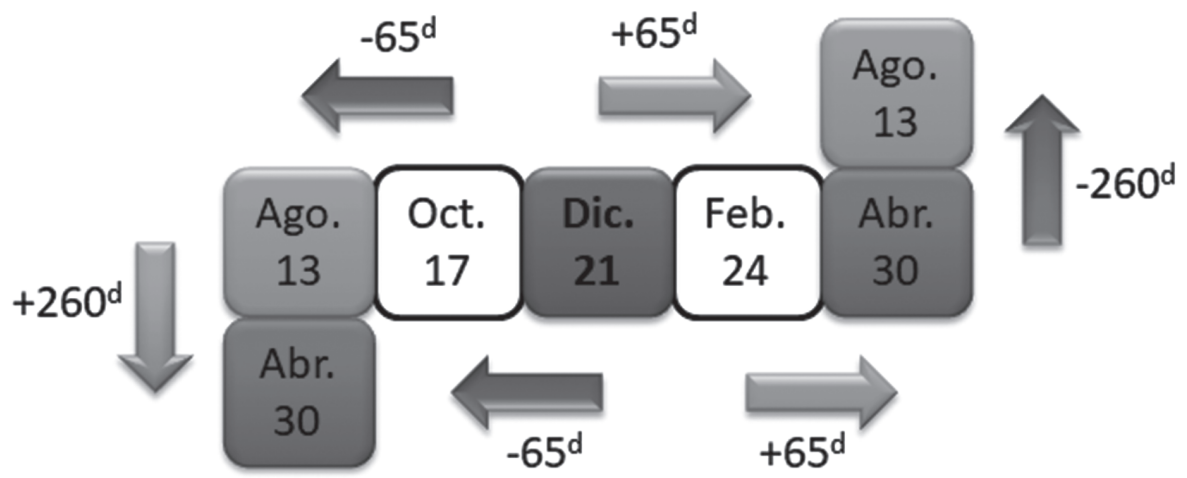

Figura 3. Desarrollo inicial de una estructura de $65^{\mathrm{d}} \times 260^{\mathrm{d}}$. Solsticio de referencia: diciembre 21 de 599, según el calendario gregoriano proléptico. Autor: Carlos Barrera Atuesta.

En un sistema reticular de estas características, cada fecha solar registrada será obtenida nuevamente después de transcurridos 118 desplazamientos horizontales consecutivos ( $c f$. Tablas 2 y 3), en virtud de que:

$$
\begin{aligned}
& B=118 \times 65^{\mathrm{d}}=7670^{\mathrm{d}} \quad A=365.242275^{\mathrm{d}} \\
& C=20 \quad r=365.154500^{\mathrm{d}} \\
& R^{\prime}=365.154500^{\mathrm{d}}-365.242275^{\mathrm{d}}=-0.087775^{\mathrm{d}}
\end{aligned}
$$

Donde $r$ y $R$ ' representan las soluciones positiva y negativa del sistema, es decir, las posiciones en adelanto y en atraso del intervalo $(B)$ múltiplo de $65^{\mathrm{d}}$, respecto del año trópico real $(A)$. Estos resultados indican, por tanto, que han transcurrido 20 años solares $(C)$, más la fracción $365.1545^{\mathrm{d}}(r)$, y que tan solo falta —pues así lo indica el signo negativo del valor de $R$ ' - una fracción de $0.087775^{\mathrm{d}}$ para completar el ciclo siguiente (el vigésimo primero).

Por ende, la diferencia acumulada entre 21 años trópicos del período Clásico (conforme era su duración para finales del siglo vi; Meeus y Savoie, 1992: 42) y 118 repeticiones de $65^{\mathrm{d}}$, corresponde a $0.087775^{\mathrm{d}}$ (2 horas, 6 minutos y 23.76 segundos). Sin embargo, la sincronía entre el ciclo ritual de $260^{\mathrm{d}}$ y el calendario solar se obtiene únicamente tras recorrer 236 celdas horizontales (ver Tablas 2 y 3), en cuyo momento la diferencia entre 42 años solares y 59 ciclos rituales es de $0.175550^{\mathrm{d}}$ ( 4 horas, 12 minutos y 47.52 segundos) ya que:

$$
\begin{aligned}
& B=236 \times 65^{\mathrm{d}}=15340^{\mathrm{d}} A=365.242275^{\mathrm{d}} \\
& C=41 \quad r=365.066725^{\mathrm{d}} \\
& R^{\prime}=365.066725^{\mathrm{d}}-365.242275^{\mathrm{d}}=-0.175550^{\mathrm{d}}
\end{aligned}
$$

Por tal motivo, la precisión del modelo (establecida en \pm 1 día) será suficiente para efectuar proyecciones confiables en un rango de \pm 239.248077 años (ya que 
se acumula un error de $\pm 1^{\mathrm{d}}$ por cada $\pm 42 / 0.175550$ años), tiempo suficiente para anticipar las condiciones climatológicas generales que determinarán los ciclos agrícolas y rituales de las siguientes nueve o diez generaciones.

Como consecuencia de las restricciones impuestas por estos sistemas de retículas que reflejan una de las formas más comunes en que los antiguos mayas estructuraban el tiempo (Aveni, Morandi y Peterson, 1995: tablas 1a y 1b), la separación existente entre los solsticios de invierno y verano, y entre las demás fechas opuestas-complementarias del sistema, será de $59 \times 65^{\mathrm{d}}=3835^{\mathrm{d}}$.

Grosso modo, el intervalo de $3835^{\mathrm{d}}$ representa el cumplimiento de $10^{1 / 2}$ años trópicos (ver Tabla 2), ya que $1 / 2 \times 365.242275^{\mathrm{d}}=182.6211375^{\mathrm{d}}$, mientras que:

$$
\begin{aligned}
& B=59 \times 65^{\mathrm{d}}=3835^{\mathrm{d}} A=365.242275^{\mathrm{d}} \\
& C=10 \quad r=182.577250^{\mathrm{d}} \\
& R^{\prime}=182.577250^{\mathrm{d}}-365.242275^{\mathrm{d}}=-182.665025^{\mathrm{d}}
\end{aligned}
$$

La estructura deducida a partir del Códice de Dresde indica, por lo tanto, que, en el contexto de un mismo ciclo solar, la separación existente entre los solsticios de invierno y verano dista muy poco de ser simétrica y mutuamente equidistante.

\section{Conjuntos de fechas de enlace}

El intervalo de separación de $3835^{\mathrm{d}}$ entre fechas opuestas-complementarias puede ser establecido con facilidad a través de dos conjuntos de "fechas de enlace" distanciadas por $7 \times 260^{\mathrm{d}}=5 \times 364^{\mathrm{d}}=1820^{\mathrm{d}}$, del entorno inmediato de ambos solsticios (o de las demás fechas que conforman el sistema).

Estos grupos de enlace (Figura 4a) se caracterizan por vincular, de forma consecutiva y alternada, fechas rituales y solares significativas coincidentes con reconocidos modelos de representación e interpretación solar basados en el estudio de orientaciones astronómicas, cámaras cenitales y calendarios de horizonte, así:

Dic. $15-1820^{\mathrm{d}}=$ Dic. $21 \quad$ Feb. $18+1820^{\mathrm{d}}=$ Feb. 12

Abr. $24-1820^{\mathrm{d}}=$ Abr. $30 \quad$ Jun. $28+1820^{\mathrm{d}}=$ Jun. 22

Febrero 12 y abril 30 pertenecen, por ejemplo, a los esquemas 1 y 2 de Šprajc (2004: 92, tabla 1), al igual que las fechas agosto 13 y octubre 30, obtenidas a partir de un segundo grupo de fechas de enlace (Figura $4 \mathrm{~b}$ ) que opera como sigue:

$$
\begin{array}{ll}
\text { Jun. } 16-1820^{d}=\text { Jun. } 22 & \text { Ago. } 20+1820^{d}=\text { Ago. } 13 \\
\text { Oct. } 24-1820^{d}=\text { Oct. } 30 & \text { Dic. } 28+1820^{d}=\text { Dic. } 21
\end{array}
$$




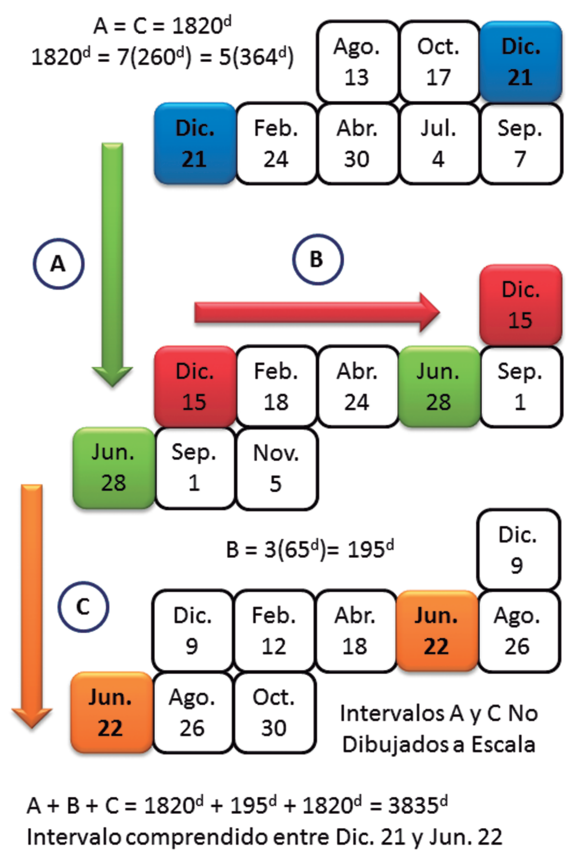

A

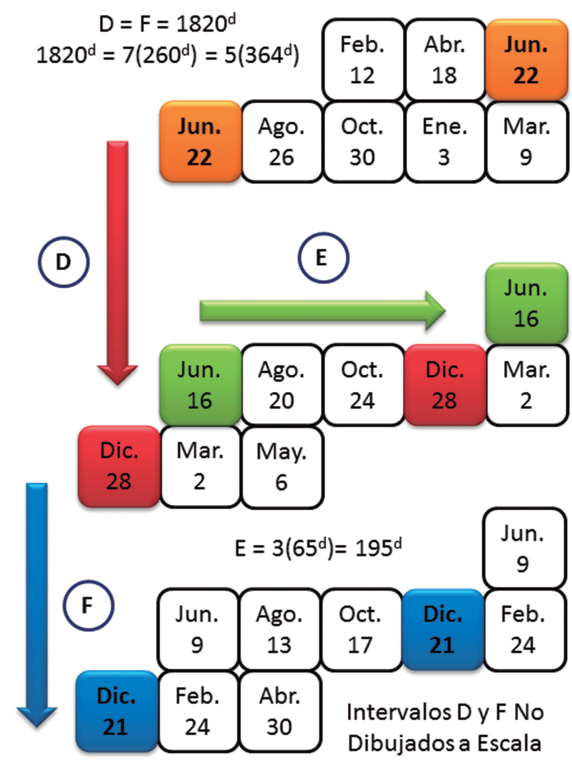

$D+E+F=1820^{d}+195^{d}+1820^{d}=3835^{d}$

Intervalo comprendido entre Jun. 22 y Dic. 21

B

Figura 4. a) Transición entre diciembre 21 (de 599) y junio 22 (de 610).

b) Transición entre junio 22 (de 610) y diciembre 21 (de 620). Autor: Carlos Barrera Atuesta.

Las demás fechas, por su parte, pertenecen a estructuras identificadas en almanaques del Códice de Dresde, coincidentes con referencias solares registradas en manuscritos coloniales como el de Juan de Rodas (Bricker, 1982: 102), en donde diciembre 28 es equiparado con la fecha 1 Tzun del calendario Tzotzil, y también con estudios más recientes de Sánchez y Šprajc (2015) que contemplan estructuras arquitectónicas orientadas hacia abril 24 y agosto 20 (Champerico, Estr. 15-a; El Tigre, Estr. 1, Edificio Superior; Tikal, Templo III; Yaxnohcah, Estr. A-1; $c f$. Figuras $4 \mathrm{a}$ y $4 \mathrm{~b}$ ), o hacia febrero 18 y octubre 23 (Tikal, Templo IV; $c f$. Figuras 4a y 4c).

En ese orden de ideas, la distancia que separa los solsticios de invierno y verano (Figura 4a) será de $1820^{\mathrm{d}}+3\left(65^{\mathrm{d}}\right)+1820^{\mathrm{d}}=3835^{\mathrm{d}}$, debido a que:

$\begin{array}{ll}\text { Dic. } 21+1820^{\mathrm{d}}=\text { Dic. } 15 & \text { Dic. } 15+3\left(65^{\mathrm{d}}\right)=\text { Jun. } 28\end{array}$

Jun. $28+1820^{\mathrm{d}}=$ Jun. $22 \quad$ Dic. $21+3835^{\mathrm{d}}=$ Jun. 22

Distancia igual a la comprendida entre los solsticios de verano e invierno (Figura $4 b)$ :

Jun. $22+1820^{\mathrm{d}}=$ Jun. $16 \quad$ Jun. $16+3\left(65^{\mathrm{d}}\right)=$ Dic. 28

Dic. $28+1820^{\mathrm{d}}=$ Dic. $21 \quad$ Jun. $22+3835^{\mathrm{d}}=$ Dic. 21 
De esta forma, se cumple un primer ciclo de $2 \times 3835^{\mathrm{d}}=7670^{\mathrm{d}}=21$ años solares, entre dichos solsticios de invierno (de 599 y 620). El solsticio de invierno de 599 ha sido seleccionado para ilustrar estas estructuras porque representa el punto medio del desarrollo del período Clásico comprendido, en términos generales, entre los años 250-950. Sin embargo, cualquier solsticio elegido arbitrariamente como referencia habrá de generar resultados similares.

Las secuencias del tipo "junio $22 \mathrm{vs}$. diciembre 21" representan las transiciones más comunes para solsticios distanciados por intervalos de $3835^{\mathrm{d}}$. No obstante lo anterior, y por motivos asociados a la forma en que opera el calendario gregoriano, la fecha diciembre 21 también puede ser obtenida eventualmente a partir de junio 21 , como efectivamente acontece $3835^{\mathrm{d}}$ antes de diciembre 21 de 599 (Figura 4c).

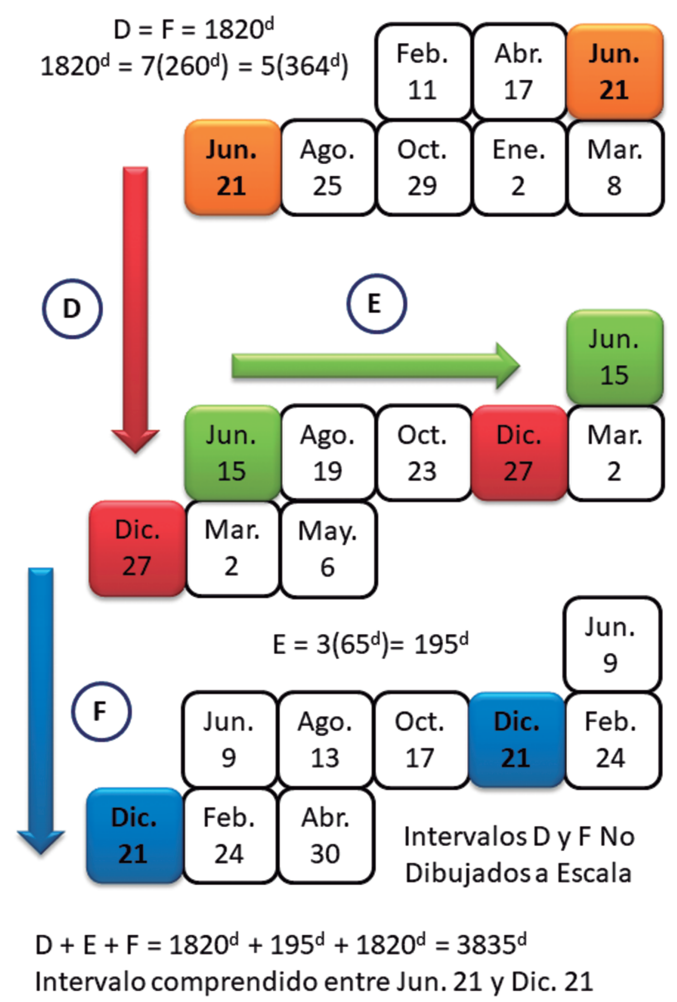

Figura 4c. Transición entre junio (de 589) y diciembre (de 599). Autor: Carlos Barrera Autesta.

A propósito, nótese la correspondencia existente entre las respectivas fechas de enlace -expresadas en función del calendario gregoriano proléptico- que preceden por $1820^{\mathrm{d}}$ al entorno de diciembre 21 de 599 (junio 15, agosto 19, 
octubre 23 y diciembre 27 de 594) y aquellas obtenidas para el año 1170 en la columna D del almanaque D.15b-16b (Figura 5; Tabla 4), que en este estudio siguen la solución de Bricker y Bricker (1992: 61, 64), pero aplicando la constante de correlación 584285 (número de días transcurridos desde las 12:00 horas de enero 1 de 4713 a.C., del calendario juliano proléptico, hasta alcanzar la Fecha Era maya 13.0.0.0.0 4 Ajaw 8 Kumk'u en septiembre 8 de 3114 a.C. del calendario juliano proléptico, equivalente a agosto 13 de 3114 a.C. del calendario gregoriano proléptico).

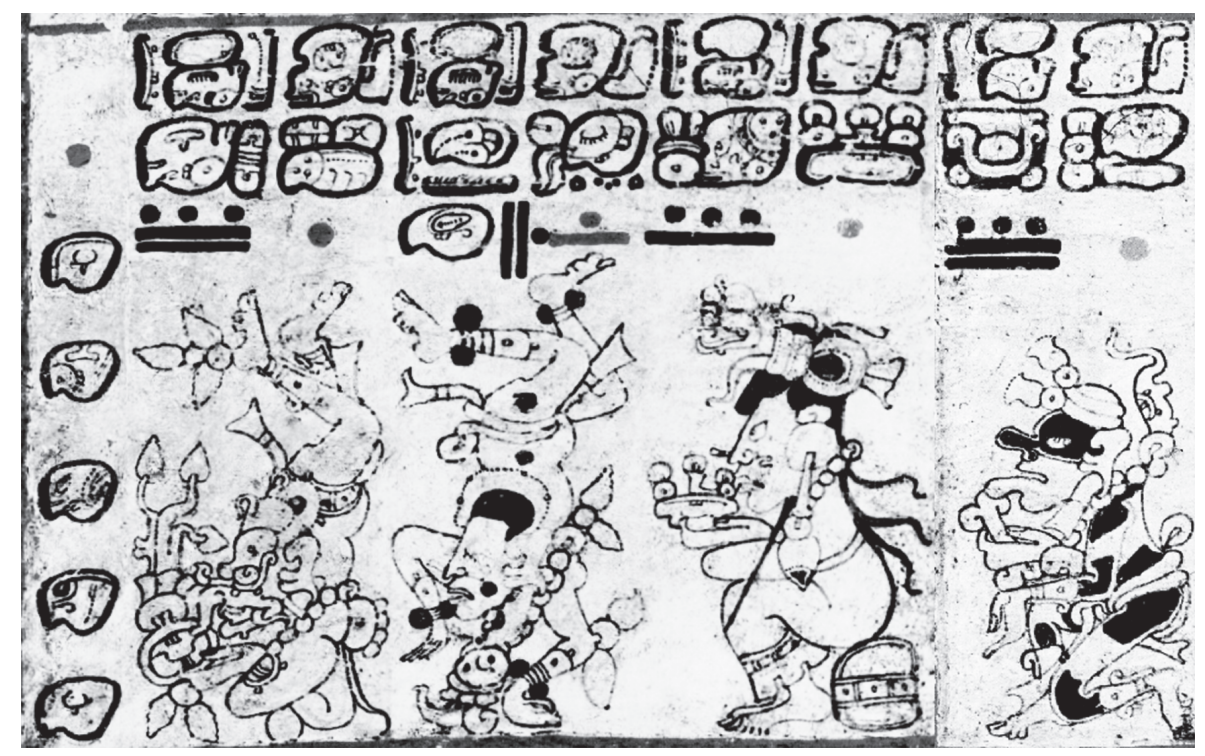

Figura 5. Almanaque D.15b-16b de 4 x $65^{\mathrm{d}}$ (Códice de Dresde).

Obsérvese asimismo la concordancia existente entre las respectivas fechas de enlace del año 605 que preceden por $1820^{\mathrm{d}}$ al entorno de junio 22 de 610 (Figura 4a), y aquellas registradas en la columna A del almanaque D.15b-16b (abril 24, junio 28 , septiembre 1 y noviembre 5 de 1170).

Respecto a estas configuraciones, existen algunos aspectos de interés que ameritan ser comentados en virtud de la importancia que reviste su contexto cultural.

El almanaque D.15b-16b y su relación con otras estructuras de $65^{\mathrm{d}}$ y $1820^{\mathrm{d}}$

El período de siembra es el tema principal del almanaque D.15b-16b, conforme lo insinúan sus escenas figurativas y lo confirman sus textos jeroglíficos. Los 
elementos iconográficos del almanaque, así como la postura singular de Chaak, asociada por los Bricker (1992) con el 'doblez del año' y la fecha 0 Yax, permitieron sugerir a dichos académicos un modelo de solución en 'tiempo real' que en su trabajo adopta la constante de correlación 584283.

\begin{tabular}{|c|c|c|c|c|c|c|c|}
\hline \multicolumn{2}{|l|}{ A } & \multicolumn{2}{|l|}{ B } & \multicolumn{2}{|l|}{ C } & \multicolumn{2}{|l|}{ D } \\
\hline $\begin{array}{l}1 \text { lk' } \\
\text { (0 Yax) } \\
10.17 .5 .1 .2 \\
\text { Abr. } 241170\end{array}$ & +13 & $\begin{array}{l}1 \text { Men } \\
(13 \text { Yax }) \\
10.17 .5 .1 .15 \\
\text { May. } 71170\end{array}$ & +31 & $\begin{array}{l}6 \text { Kimi } \\
(4 \mathrm{Kej}) \\
10.17 .5 .3 .6 \\
\text { Jun. } 71170\end{array}$ & +8 & $\begin{array}{l}1 \text { Ix } \\
(12 \text { Kej) } \\
10.17 .5 .3 .14 \\
\text { Jun. } 151170\end{array}$ & +13 \\
\hline $\begin{array}{l}\text { 1 Manik' } \\
\text { (5 Mak) } \\
10.17 .5 .4 .7 \\
\text { Jun. } 281170\end{array}$ & +13 & $\begin{array}{l}\text { 1 Ajaw } \\
\text { (18 Mak) } \\
10.17 .5 .5 .0 \\
\text { Jul. } 111170\end{array}$ & +31 & $\begin{array}{l}6 \text { Chuwen } \\
\text { (9 Muwan) } \\
\text { 10.17.5.6.11 } \\
\text { Ago. } 111170\end{array}$ & +8 & $\begin{array}{l}1 \text { Kawak } \\
\text { (17 Muwan) } \\
10.17 .5 .6 .19 \\
\text { Ago. } 191170\end{array}$ & +13 \\
\hline $\begin{array}{l}1 \text { Eb' } \\
\text { (10 Pax) } \\
10.17 .5 .7 .12 \\
\text { Sep. } 11170\end{array}$ & +13 & $\begin{array}{l}\text { 1 Chikchan } \\
\text { (3 K'ayab') } \\
10.17 .5 .8 .5 \\
\text { Sep. } 141170\end{array}$ & +31 & $\begin{array}{l}6 \text { Kib' } \\
\text { (14 Kumk'u) } \\
10.17 .5 .9 .16 \\
\text { Oct. } 151170\end{array}$ & +8 & $\begin{array}{l}1 \text { K'an } \\
\text { (2 Wayeb') } \\
10.17 .5 .10 .4 \\
\text { Oct. } 231170\end{array}$ & +13 \\
\hline $\begin{array}{l}1 \text { Kab'an } \\
\text { (10 Pop) } \\
10.17 .5 .10 .17 \\
\text { Nov. } 51170\end{array}$ & +13 & $\begin{array}{l}1 \text { Ok } \\
(3 \mathrm{Wo}) \\
10.17 .5 .11 .10 \\
\text { Nov. } 181170\end{array}$ & +31 & $\begin{array}{l}6 \text { Imix } \\
\text { (14 Sip) } \\
10.17 .5 .13 .1 \\
\text { Dic. } 191170\end{array}$ & +8 & $\begin{array}{l}1 \text { Muluk } \\
(2 \text { Sotz') } \\
10.17 .5 .13 .9 \\
\text { Dic. } 271170\end{array}$ & +13 \\
\hline
\end{tabular}

Tabla 4. Fechas de enlace en la estructura del almanaque D.15b-16b. (Calendario gregoriano proléptico, constante de correlación 584285). Autor: Carlos Barrera Atuesta.

En la versión propuesta por Victoria y Harvey Bricker, la iconografía del almanaque concuerda exclusivamente con la primera fila de la estructura (fechas comprendidas entre abril y junio de 1170), lo que excluye de su "instantánea" (en su acepción de "fotografía") a otras componentes del almanaque.

La identificación de un sistema de fechas solares que, de manera análoga a la fecha "asiento de Yax", alias 0 Yax, también representa un punto de inflexión situado a medio camino entre solsticios de tipo opuesto distanciados por $3835^{\mathrm{d}}$ permite ofrecer aquí una visión más amplia de la escena, y esclarecer un poco más su relación con otras estructuras similares.

La así denominada Tabla del Agua, comprendida entre las páginas 69 y 74 del Códice de Dresde, incluye en su sección inferior (D.70b-73c) una tabla de múltiplos de $1820^{\mathrm{d}}$ conformada por segmentos internos de $65^{\mathrm{d}}$. Aparte de sus evidentes propiedades matemáticas que permiten sincronizar siete ciclos rituales de $260^{\mathrm{d}}$ con cinco "años computacionales" de $364^{\mathrm{d}}$, poco más podía decirse acerca de las potenciales funciones de este ciclo de $1820^{\mathrm{d}}$.

En el contexto del almanaque D.15b-16b, sendos intervalos - negativo y positivo- de $1820 \mathrm{~d}$ vinculan las fechas solares abril 24 y noviembre 5 (Tabla 4) 
— localizadas al inicio y al final de la columna A de la estructura — con las fechas canónicas abril 30 y octubre 30 (cf. Figuras $4 \mathrm{a}$ y $4 \mathrm{~b}$; Tablas 5 y 6) —que designan el inicio y el final de la temporada de lluvias en extensas regiones de Mesoamérica- estableciendo de esta forma una relación de afinidad con el argumento central de la Tabla del Agua. Nótese la correspondencia cercana respecto a las proyecciones de $1820^{\mathrm{d}}$ y $65^{\mathrm{d}}$ de Bricker y Bricker (2011: 398s) —donde 9.16.18.4.7 es mayo 1 de 769—y Vail y Hernández (2013: 155s) —donde 9.5.6.7.7 es octubre 28 de 540 - así como la concordancia iconográfica de la cifra 5.1 .0 de $1820^{\mathrm{d}}$ en el agua vertida por Chak Chel desde su cántaro durante la temporada de lluvias (Códice de Dresde, 74).

Por su parte, los datos obtenidos en la columna D de la estructura (Tabla 4) enlazan el solsticio de verano del año 1165 (localizado $1820^{\mathrm{d}}$ antes de junio 15 de 1170; Tabla 5), con el solsticio de invierno del año 1175 (localizado $1820^{\text {d }}$ después de diciembre 27 de $1170 ; c f$. Tabla 6 ), mediante un intervalo de $1820^{\mathrm{d}}+$ $3\left(65^{\mathrm{d}}\right)+1820^{\mathrm{d}}=3835^{\mathrm{d}}$, generándose de este modo una relación de correspondencia entre pares opuestos-complementarios (Figura 4c).

\begin{tabular}{|c|c|c|c|c|c|c|c|}
\hline \multicolumn{2}{|l|}{ A } & \multicolumn{2}{|l|}{ B } & \multicolumn{2}{|l|}{ C } & \multicolumn{2}{|l|}{ D } \\
\hline $\begin{array}{l}1 \text { lk' } \\
\text { (5 Yax) } \\
10.17 .0 .0 .2 \\
\text { Abr. } 301165\end{array}$ & +13 & $\begin{array}{l}1 \text { Men } \\
(18 \text { Yax }) \\
10.17 .0 .0 .15 \\
\text { May. } 131165\end{array}$ & +31 & $\begin{array}{l}6 \text { Kimi } \\
\text { (9 Kej) } \\
10.17 .0 .2 .6 \\
\text { Jun. } 131165\end{array}$ & +8 & $\begin{array}{l}1 \mathrm{Ix} \\
(17 \mathrm{Kej}) \\
10.17 .0 .2 .14 \\
\text { Jun. } 211165\end{array}$ & +13 \\
\hline $\begin{array}{l}\text { 1 Manik' } \\
\text { (10 Mak) } \\
10.17 .0 .3 .7 \\
\text { Jul. } 41165\end{array}$ & +13 & $\begin{array}{l}\text { 1 Ajaw } \\
\text { (3 K'ank'in) } \\
10.17 .0 .4 .0 \\
\text { Jul. } 171165\end{array}$ & +31 & $\begin{array}{l}6 \text { Chuwen } \\
\text { (14 Muwan) } \\
\text { 10.17.0.5.11 } \\
\text { Ago. } 171165\end{array}$ & +8 & $\begin{array}{l}1 \text { Kawak } \\
(2 \text { Pax }) \\
\text { 10.17.0.5.19 } \\
\text { Ago. } 251165\end{array}$ & +13 \\
\hline $\begin{array}{l}1 \text { Eb' } \\
\text { (15 Pax) } \\
10.17 .0 .6 .12 \\
\text { Sep. } 71165\end{array}$ & +13 & $\begin{array}{l}\text { 1 Chikchan } \\
\text { (8 K'ayab') } \\
10.17 .0 .7 .5 \\
\text { Sep. } 201165\end{array}$ & +31 & $\begin{array}{l}6 \text { Kib' } \\
\text { (19 Kumk'u) } \\
10.17 .0 .8 .16 \\
\text { Oct. } 211165\end{array}$ & +8 & $\begin{array}{l}1 \text { K'an } \\
\text { (2 Pop) } \\
\text { 10.17.0.9.4 } \\
\text { Oct. } 291165\end{array}$ & +13 \\
\hline $\begin{array}{l}1 \text { Kab'an } \\
\text { (15 Pop) } \\
10.17 .0 .9 .17 \\
\text { Nov. } 11 \text { 1165 }\end{array}$ & +13 & 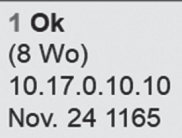 & +31 & $\begin{array}{l}6 \text { Imix } \\
\text { (19 Sip) } \\
10.17 .0 .12 .1 \\
\text { Dic. } 251165\end{array}$ & +8 & $\begin{array}{l}1 \text { Muluk } \\
\text { (7 Sotz') } \\
10.17 .0 .12 .9 \\
\text { Ene. } 21166\end{array}$ & +13 \\
\hline
\end{tabular}

Tabla 5. Fechas canónicas de 1165-6 en columnas A y D del almanaque D.15b-16b. (Calendario gregoriano proléptico, constante de correlación 584285; cf. Figuras 3, 4a y 4c.) Autor: Carlos Barrera Atuesta

\section{Los registros cronológicos del sitio clásico de Machaquilá, Petén, Guatemala}

Una serie de inscripciones cronológicas del asentamiento arqueológico de Machaquilá — situado en la región suroriental de Petén, Guatemala - conmemoran la culminación de períodos ho'tuun de 5 x 360 , y evidencian el seguimiento simultáneo de los tres principales ciclos solares mayas $\left(360^{\mathrm{d}}, 364^{\mathrm{d}}\right.$ y $\left.365^{\mathrm{d}}\right)$ mediante el registro de intervalos de $1800^{\mathrm{d}}, 1820^{\mathrm{d}}$ y $1825^{\mathrm{d}}$ (Iwaniszewski, 2013: 300, Tabla 6). 


\begin{tabular}{|c|c|c|c|c|c|c|c|}
\hline \multicolumn{2}{|l|}{ A } & \multicolumn{2}{|l|}{ B } & \multicolumn{2}{|l|}{ C } & \multicolumn{2}{|l|}{ D } \\
\hline $\begin{array}{l}1 \text { l lk' } \\
\text { (15 Ch'en) } \\
10.17 .10 .2 .2 \\
\text { Abr. } 181175\end{array}$ & +13 & $\begin{array}{l}1 \text { Men } \\
(8 \text { Yax }) \\
10.17 .10 .2 .15 \\
\text { May. } 11175\end{array}$ & +31 & $\begin{array}{l}6 \text { Kimi } \\
\text { (19 Sak) } \\
\text { 10.17.10.4.6 } \\
\text { Jun. 1 1175 }\end{array}$ & +8 & \begin{tabular}{|l}
1 Ix \\
$(7 \mathrm{Kej})$ \\
10.17 .10 .4 .14 \\
Jun. 91175
\end{tabular} & +13 \\
\hline $\begin{array}{l}\text { 1 Manik' } \\
\text { (O Mak) } \\
10.17 .10 .5 .7 \\
\text { Jun. } 221175\end{array}$ & +13 & \begin{tabular}{|l} 
1 Ajaw \\
(13 Mak) \\
10.17 .10 .6 .0 \\
Jul. 5 1175 \\
\end{tabular} & +31 & $\begin{array}{l}6 \text { Chuwen } \\
\text { (4 Muwan) } \\
\text { 10.17.10.7.11 } \\
\text { Ago. } 51175\end{array}$ & +8 & $\begin{array}{l}\text { 1 Kawak } \\
\text { (12 Muwan) } \\
\text { 10.17.10.7.19 } \\
\text { Ago. } 131175\end{array}$ & +13 \\
\hline $\begin{array}{l}1 \text { Eb' } \\
\text { (5 Pax) } \\
10.17 .10 .8 .12 \\
\text { Ago. } 261175\end{array}$ & +13 & $\begin{array}{l}\text { Chikchan } \\
\text { (18 Pax) } \\
10.17 .10 .9 .5 \\
\text { Sep. } 81175\end{array}$ & +31 & $\begin{array}{l}6 \text { Kib' } \\
\text { (9 Kumk'u) } \\
10.17 .10 .10 .16 \\
\text { Oct. } 91175\end{array}$ & +8 & $\begin{array}{l}\text { 1 K'an } \\
\text { (17 Kumk'u) } \\
10.17 .10 .11 .4 \\
\text { Oct. } 171175\end{array}$ & +13 \\
\hline $\begin{array}{l}1 \text { Kab'an } \\
\text { (5 Pop) } \\
10.17 .10 .11 .17 \\
\text { Oct. } 301175\end{array}$ & +13 & \begin{tabular}{|l} 
Ok \\
(18 Pop) \\
10.17 .10 .12 .10 \\
Nov. 121175
\end{tabular} & +31 & $\begin{array}{l}6 \text { Imix } \\
(9 \text { Sip) } \\
10.17 .10 .14 .1 \\
\text { Dic. } 131175\end{array}$ & +8 & \begin{tabular}{|l} 
Muluk \\
(17 Sip) \\
10.17 .10 .14 .9 \\
Dic. 211175
\end{tabular} & +13 \\
\hline
\end{tabular}

Tabla 6. Fechas canónicas de 1175 en columnas A y D del almanaque D.15b-16b.

(Calendario gregoriano proléptico, constante de correlación 584285; cf. Figuras 3, 4a y 4b.) Autor: Carlos Barrera Atuesta

En las estelas bajo estudio (Tabla 7), el cumplimiento de los intervalos de $5 \mathrm{x}$ $364^{\mathrm{d}}=7 \times 260^{\mathrm{d}}=1820^{\mathrm{d}}$ coincide con fechas de dedicación 1 Ajaw de la cuenta ritual, y los ciclos de $5 \times 365^{\mathrm{d}}=1825^{\mathrm{d}}$ concuerdan con registros 13 Kumk'u del calendario Jaab'. Para facilitar su identificación en la Tabla 7, las fechas 1 Ajaw han sido destacadas en rojo, y las dataciones 13 Kumk'u, en verde.

\begin{tabular}{|c|c|c|}
\hline Monumento & Fin de Período & Fecha de Dedicación \\
\hline Estela 18 & $\begin{array}{l}\text { 9.17.5.0.0 } 6 \text { Ajaw } 13 \text { K'ayab' } \\
\text { Dic. } 29775\end{array}$ & $\begin{array}{l}\text { 9.17.5.1.0 13 Ajaw } 13 \text { Kumk'u } \\
\text { Ene. } 18776\end{array}$ \\
\hline Estela 2 & $\begin{array}{l}\text { 9.18.10.0.0 10 Ajaw } 8 \text { Sak } \\
\text { Ago. } 19800\end{array}$ & $\begin{array}{l}\text { 9.18.10.7.5 } 12 \text { Chikchan } 13 \text { Kumk'u } \\
\text { Ene. } 11801\end{array}$ \\
\hline Estela 3 & $\begin{array}{l}\text { 9.19.5.0.0 } 2 \text { Ajaw } 13 \text { Yaxk'in } \\
\text { Jun. } 2815\end{array}$ & $\begin{array}{l}\text { 9.19.5.11.0 } 1 \text { Ajaw } 13 \text { Kumk'u } \\
\text { Ene. } 8 \text { 816 }\end{array}$ \\
\hline Estela 4 & $\begin{array}{l}\text { 9.19.10.0.0 8 Ajaw } 8 \mathrm{Xul} \\
\text { May. } 6820\end{array}$ & $\begin{array}{l}\text { 9.19.10.12.0 } 1 \text { Ajaw } 8 \text { Kumk'u } \\
\text { Ene. } 1821\end{array}$ \\
\hline Estela 8 & $\begin{array}{l}\text { 9.19.15.0.0 } 1 \text { Ajaw } 3 \text { Sek } \\
\text { Abr. } 10825\end{array}$ & $\begin{array}{l}\text { 9.19.15.13.0 } 1 \text { Ajaw } 3 \text { Kumk'u } \\
\text { Dic. } 26825\end{array}$ \\
\hline Estela 7 & $\begin{array}{l}\text { 10.0.0.0.0 } 7 \text { Ajaw } 18 \mathrm{Sip} \\
\text { Mar. } 15830\end{array}$ & $\begin{array}{l}\text { 10.0.0.14.15 } 3 \text { Men } 13 \text { Kumk'u } \\
\text { Ene. } 4831\end{array}$ \\
\hline Estela 6 & $\begin{array}{l}\text { 10.0.5.0.0 13 Ajaw } 13 \text { Wo } \\
\text { Feb. } 17835\end{array}$ & $\begin{array}{l}\text { 10.0.5.16.0 8 Ajaw } 13 \text { Kumk'u } \\
\text { Ene. } 3836\end{array}$ \\
\hline Estela 5 & $\begin{array}{l}\text { 10.0.10.0.0 } 6 \text { Ajaw } 8 \text { Pop } \\
\text { Ene. } 22840\end{array}$ & $\begin{array}{l}\text { 10.0.10.17.5 } 13 \text { Chikchan } 13 \text { Kumk'u } \\
\text { Ene. } 1841\end{array}$ \\
\hline
\end{tabular}

Tabla 7. Registros cronológicos de Machaquilá.

Basado en Iwaniszewski (2013: 300, Tabla 6). 
Otros intervalos representativos comprendidos entre las inscripciones cronológicas de Machaquilá están constituidos por ciclos mayas reconocidos no contemplados en estudios previos que, en último análisis, inducen a reconsiderar la forma en que pudo haber sido establecida la sincronía entre ciclos rituales y solares.

Las fechas 6 Ajaw de las Estelas 18 y 5 -en color púrpura- representan una transición de $23400^{\mathrm{d}}$, conformada por cinco repeticiones del intervalo maya de $4680^{\mathrm{d}}$ (Códice de Dresde, 58b y 59c) utilizado para proyectar eclipses de tipo opuesto, y para rastrear los movimientos sinódicos de Venus y Mercurio. Dicho intervalo de separación de $23400^{\mathrm{d}}$, se encuentra sugerido además entre el fin de k'atun 9.17.0.0.0 — a partir del cual se habrían efectuado sendas transiciones de $1800 \mathrm{~d}$ y $1820 \mathrm{~d}$ hacia la Estela 18 - y la fecha 10.0.5.0.0 de la Estela 6.

La convergencia de ciclos de $1820^{\mathrm{d}}$ y $1825^{\mathrm{d}}$ sobre la fecha de dedicación de la Estela 3 resalta la importancia que habría tenido la Rueda Calendárica 1 Ajaw 13 Kumk'u para los antiguos habitantes de Machaquilá. Por tal motivo, resulta lógico suponer que los integrantes de su élite practicaran el seguimiento de dichas componentes rituales y Jaab' a través del ciclo de $73 \times 260^{\mathrm{d}}=52 \times 365^{\mathrm{d}}=$ $18980^{\mathrm{d}}$, atestiguado en las páginas 65-72,73b del Códice de Madrid (Vail y Aveni, 2004: 172s), y asociado en otros contextos culturales de la antigua Mesoamérica con las ceremonias del Fuego Nuevo.

Otro período notable, esta vez establecido de manera explícita entre las respectivas fechas de dedicación de las Estelas 3 y 8 , describe una duración de 2 x $1820^{\mathrm{d}}=3640^{\mathrm{d}}$ ( $c f$. Códice de Dresde, 45a, 64a y 71e). En tanto que las Estelas 4 y 5 , dedicadas con veinte años de diferencia, se correlacionan con la misma fecha solar de enero 1. Este hecho sugiere, tal y como lo había inferido Broda (1983), que en la antigua Mesoamérica ya existían métodos para estimar la duración del año solar en, al menos, $365^{1 / 4^{d}}$ (resultado que se obtiene al analizar el intervalo de $7305^{\mathrm{d}}$ comprendido entre las fechas de dedicación de las Estelas 4 y 5 de Machaquilá), y que este conocimiento pudo haber sido aplicado para intentar la coordinación entre ritos, fenómenos climatológicos periódicos y ciclos agrícolas.

La orientación de estructuras arquitectónicas hacia puntos específicos del horizonte habría resuelto efectivamente las dificultades para fijar fechas solares, pero no el asunto de la sincronía entre ciclos solares y rituales. Se concluye, por tanto, que "otro método, aún desconocido", debió ser utilizado, y que se requiere revisar con atención la información aquí argüida para procurar dilucidarlo.

\section{Ciclos rituales-solares}

De la discusión anterior, podría extraerse lo siguiente:

- Un intervalo de $18980^{d}$ debió ser aplicado desde la fecha de dedicación de la Estela 3 para dar continuidad, y hacer seguimiento de la Rueda Calendárica 1 Ajaw 13 Kumk'u. 
- Un intervalo de $3640^{\mathrm{d}}$ fue evidentemente aplicado sobre la misma fecha de dedicación de la Estela 3, motivando la respectiva inscripción de la Estela 8.

- Debió existir un método para determinar la posición que ocupaban las fechas registradas respecto del año trópico; hipotéticamente, un rasgo destacado del paisaje en donde el sol emergía (o se ocultaba) en dichos tiempos específicos del año (el método utilizado no es relevante para el análisis; las fechas inscritas sí lo son).

Los antiguos mayas eran astrónomos avezados, así que debieron proceder a registrar la posición del sol en el horizonte para la fecha de dedicación de la Estela 3 (enero 8, según la información suministrada por la Tabla 7). La fecha de dedicación de la Estela 8, conforme consta en la Tabla 7, es equivalente a diciembre 26 (según el calendario gregoriano proléptico); punto que los antiguos astrónomos mayas también habrían señalado en el horizonte.

Resta por establecer entonces la fecha solar alcanzada después de transcurridos $18980^{\mathrm{d}}$ desde la fecha de dedicación de la Estela 3. Para este fin, podríamos fijar un módulo aritmético de $365.242253^{\mathrm{d}}$ (duración del año trópico para mediados del siglo ix; ver Tabla 2; $c f$. Meeus and Savoie, 1992: 42), así:

$$
\begin{aligned}
& (18980 \mathrm{~d}) \bmod \left(365.242253^{\mathrm{d}}\right)=352.645097^{\mathrm{d}} \quad \text { 'solución positiva' } \\
& 352.645097^{\mathrm{d}}-365.242253^{\mathrm{d}}=-12.597156^{\mathrm{d}} \quad \text { 'solución negativa' }
\end{aligned}
$$

Por lo tanto, se requiere retroceder $12.597156 \mathrm{~d}$ desde enero 8 (fecha de dedicación de la Estela 3) para conocer la fecha que habrían atestiguado los antiguos mayas en el horizonte una vez completada la siguiente Rueda Calendárica 1 Ajaw 13 Kumk'u. La sustracción preliminar de 12.0d desde enero 8 conduce hacia diciembre 27, así que la sustracción de la fracción restante de día (0.597156) -que aproximaremos a 1.0 porque los antiguos mayas solo manejaban números enteros- habría conducido en consecuencia hacia diciembre 26. Es decir, hacia la misma posición solar registrada en la inscripción de la Estela 8.

Para sorpresa del astrónomo ancestral que hubiese efectuado estas observaciones, no solo habrían coincidido las respectivas posiciones del sol en el horizonte para la recién obtenida Rueda Calendárica y para la fecha de dedicación de la Estela 8, sino que también habrían coincidido sus respectivos componentes rituales. Un importante intervalo de sincronía entre ciclos solares y rituales había sido descubierto, y su duración era inferior a la expectativa de vida de muchos gobernantes mayas de la época (Martin y Grube, 2000), de tal suerte que el cumplimiento de dos de estos ciclos consecutivos de sincronización bien pudo haber sido atestiguado por un mismo individuo, y por sus descendientes directos.

El intervalo obtenido corresponde en consecuencia a $18980^{\mathrm{d}}-3640^{\mathrm{d}}=$ $15340^{\mathrm{d}}$, y puede ser expresado en función, tanto del ciclo ritual, como del año solar, a través de la relación de correspondencia $59 \times 260^{\mathrm{d}}=42$ años solares. 
Para facilitar los cómputos cronológicos, las Figuras 6-9 incorporan un identificador único de fechas técnicamente denominado ‘día juliano' (DJ), que representa la cuenta correlativa de días transcurridos desde el mediodía de enero 1 de 4713 a.C., según el calendario juliano proléptico. De esta forma, el lector podrá adicionar o sustraer los intervalos indicados de sus respectivos días julianos. Debe tenerse presente, sin embargo, que las demás fechas ilustradas en dichas imágenes continúan siendo expresadas en función del calendario gregoriano.

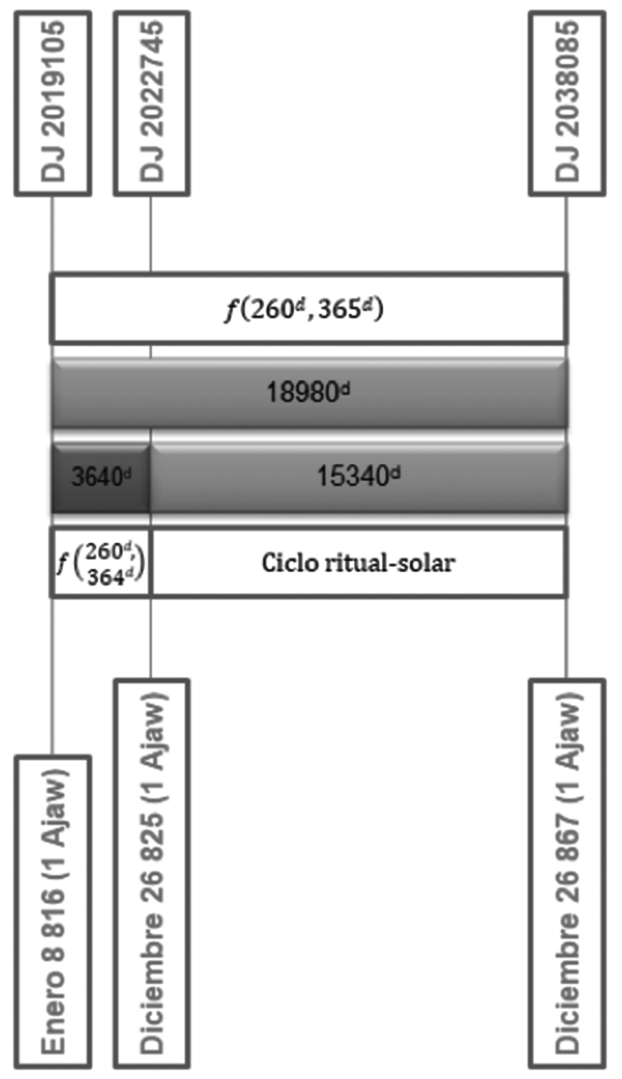

Figura 6. Proyección de la fecha diciembre 26 de 825 (cf. Tabla 7) a través de un ciclo ritual-solar de $15340 \mathrm{~d}=18980 \mathrm{~d}-3640 \mathrm{~d}$.

Autor: Carlos Barrera Atuesta

El análisis de los intervalos ilustrados en la Figura 6 desvela una propiedad inédita de los múltiplos de $1820^{\mathrm{d}}$ que se traduce en su capacidad para anticipar los efectos que genera la Rueda Calendárica sobre la posición del sol en el horizonte y, por consiguiente, en la fijación de fechas solares. Por tal motivo, la fecha obtenida después de transcurridos 52 ciclos de 365d, i.e. 18980d, será la misma 
fecha obtenida después de cumplidos tan solo 10 ciclos de $364^{\mathrm{d}}$, i.e. $3640^{\mathrm{d}}=2$ x $1820^{\text {d }}$.

Por extensión, los mayas habrían podido deducir entonces que el efecto solar generado por 104 ciclos de $365^{\mathrm{d}}$, i.e. $37960^{\mathrm{d}}$, equivalentes a 65 ciclos canónicos de Venus de $584^{\mathrm{d}}$ (Códice de Dresde, 24, 46-50), era el mismo generado por tan solo 20 ciclos de $364^{\mathrm{d}}$, i.e. $7280^{\mathrm{d}}$, equivalentes a 4 ciclos de $1820^{\mathrm{d}}$ (Códice de Dresde, 64), y que, en último análisis, esto era debido a que la diferencia entre sus respectivas duraciones concordaba con el cumplimiento de 84 años solares, es decir, con dos ciclos rituales-solares de $15340^{\mathrm{d}}$ (ver Figura 7).

$\left(104 \times 365^{\mathrm{d}}\right)-\left(20 \times 364^{\mathrm{d}}\right)=30680^{\mathrm{d}}=2 \times 15340^{\mathrm{d}}=84$ años solares

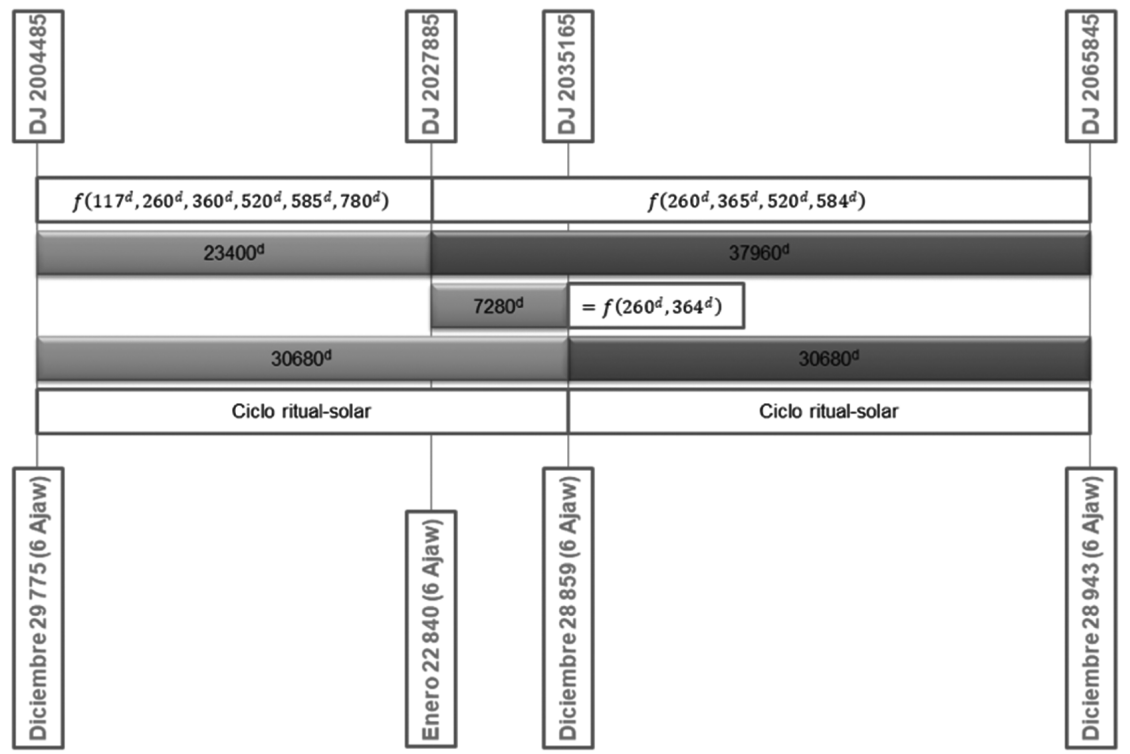

Figura 7. Obtención de ciclos rituales-solares de $30680^{\mathrm{d}}$ a partir de estructuras básicas de $7280^{\mathrm{d}}, 23400^{\mathrm{d}}$ y $37960^{\mathrm{d}}$ ( $c f$. Tabla 7).

Autor: Carlos Barrera Atuesta

Es en este punto del análisis donde adquiere sentido práctico el intervalo de $23400^{\mathrm{d}}$ identificado entre las inscripciones cronológicas de las Estelas 18 y 5 de Machaquilá, ya que dicho período (equivalente a 65 ciclos de $360^{\mathrm{d}}$ ) puede ser complementado con una veintena de 'años computacionales' de $364^{\mathrm{d}}$ para producir, nuevamente, 84 años solares (Tabla 7; Figura 7).

$\left(65 \times 360^{\mathrm{d}}\right)+\left(20 \times 364^{\mathrm{d}}\right)=30680^{\mathrm{d}}=2 \times 15340^{\mathrm{d}}=84$ años solares

Esto explicaría, además, por qué resultaba esencial para la antigua élite de Machaquilá realizar el seguimiento simultáneo de los ciclos solares fundamentales de $360^{\mathrm{d}}, 364^{\mathrm{d}}$ y $365^{\mathrm{d}}$. 


\section{Intervalos congruentes}

La revisión de los casos anteriores permite proponer un procedimiento sencillo para obtener intervalos canónicos congruentes, es decir, intervalos que generan desplazamientos similares respecto del ciclo anual, tal y como acontece con los intervalos de $7280^{\mathrm{d}}, 37960^{\mathrm{d}}$ y $-23400^{\mathrm{d}}$ (ver Tablas 2 y 3 ).

$$
\begin{array}{ll}
\left(7280^{\mathrm{d}}\right) \bmod \left(365.242253^{\mathrm{d}}\right) & R^{\prime}=-24.845060^{\mathrm{d}} \\
\left(37960^{\mathrm{d}}\right) \bmod \left(365.242253^{\mathrm{d}}\right) & R^{\prime}=-25.194312^{\mathrm{d}} \\
\left(-23400^{\mathrm{d}}\right) \bmod \left(365.242253^{\mathrm{d}}\right) & R^{\prime}=-24.495808^{\mathrm{d}}
\end{array}
$$

El procedimiento en cuestión consiste en adicionar o sustraer múltiplos consecutivos de $118 \times 65^{\mathrm{d}}=7670^{\mathrm{d}}$ a partir de un ciclo maya reconocido hasta lograr obtener otro intervalo canónico significativo. Para el caso aquí referido, los intervalos de $37960^{\mathrm{d}} \mathrm{y}-23400^{\mathrm{d}}$ habrían sido sintetizados a partir del ciclo de $7280^{\mathrm{d}}=$ $28 \times 260^{\mathrm{d}}=20 \times 364^{\mathrm{d}}=4 \times 1820^{\mathrm{d}}$, de la siguiente forma:

$$
\begin{aligned}
& 7280^{d}+4\left(7670^{d}\right)=37960^{d} \\
& 7280^{d}-4\left(7670^{d}\right)=-23400^{d}
\end{aligned}
$$

Se considera que los intervalos de $37960^{\mathrm{d}}$ y $23400^{\mathrm{d}}$ son significativos porque, al igual que el intervalo de $7280^{\mathrm{d}}$, pueden ser expresados en función de ciclos mayas reconocidos, como $117^{\mathrm{d}}, 260^{\mathrm{d}}, 360^{\mathrm{d}}, 365^{\mathrm{d}}, 584^{\mathrm{d}}$ y $585^{\mathrm{d}}$. Condición que aquí se cumple, ya que $37960^{\mathrm{d}}$ es función de $260^{\mathrm{d}}, 365^{\mathrm{d}}$ y $584^{\mathrm{d}}$; mientras $23400^{\mathrm{d}}$ es función de $117^{\mathrm{d}}, 360^{\mathrm{d}}$ y $585^{\mathrm{d}}$ (ver Figura 7 ).

Por otra parte, los intervalos múltiplos de $7670^{\mathrm{d}}$ fueron estudiados bajo diferentes circunstancias por al menos cinco investigadores, que aportaron evidencia y sustento teórico. En 1981, Robert D. Peden ${ }^{2}$ propuso un sistema de enclavamiento de ciclos celestes en donde 42 años trópicos eran equiparados con 59 ciclos rituales de 260 ; en 1995 Elzbieta Siarkiewicz deduce a partir de códices no mayas que 42 años trópicos son equivalentes a 15340d; en 2000, Simon Martin y Nikolai Grube advierten que sendas inscripciones epigráficas registradas en los Monumentos 161, 3, 29, 111 y 134 de Toniná (Mathews, 2001) se encuentran distanciadas por 59 ciclos rituales de $260^{\mathrm{d}}$ que describen 42 años solares; y en 2011 , el autor descubre que el intervalo de $30680^{d}$ representa la sincronía entre 84 años solares, 118 ciclos rituales y 83 períodos sinódicos de Urano (el planeta más distante aún detectable a simple vista desde la Tierra bajo condiciones atmosféricas óptimas).

El intervalo de $30680^{\mathrm{d}}=11960^{\mathrm{d}}+2\left(9360^{\mathrm{d}}\right)$ también posee el potencial necesario para vincular durante su trayecto diversas zonas de eclipses, conforme se deduce de la anterior identidad expresada en función de ciclos mayas de $11960^{\mathrm{d}}$ y $9360^{\mathrm{d}}$, usados para describir dicho tipo de eventos.

${ }^{2}$ Documento HTML de 2004 [1981] en posesión del suscrito autor. 


\section{Utilidad práctica}

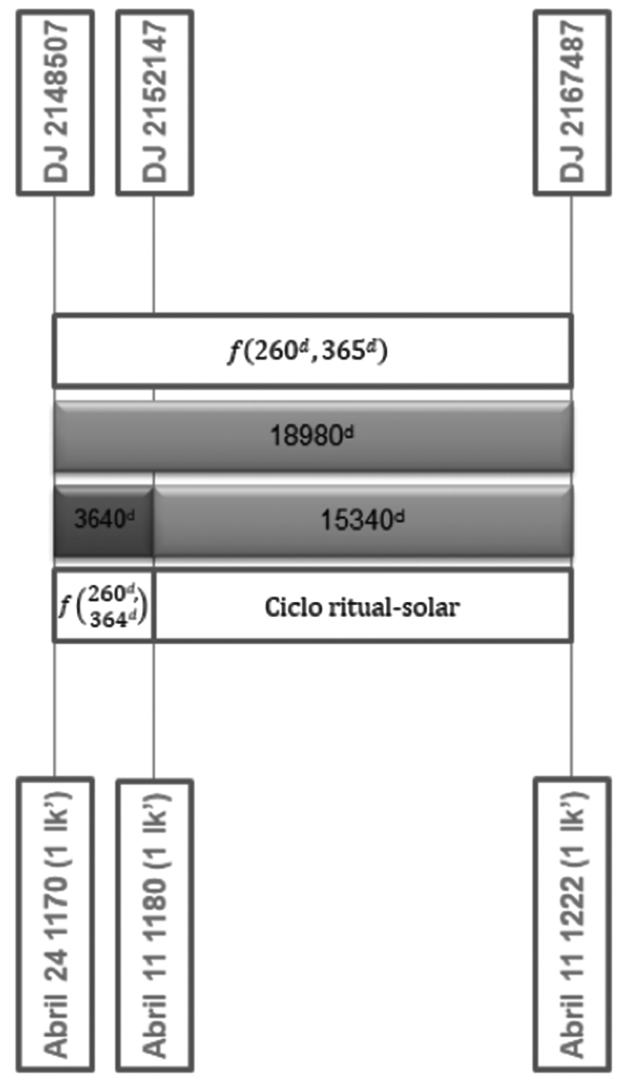

Figura 8. Proyección de la fecha abril 11 de 1180 ( $c f$. Tablas 4,8 y 9) a través de un ciclo ritual-solar de $15340^{\mathrm{d}}=18980^{\mathrm{d}}-3640^{\mathrm{d}}$.

Autor: Carlos Barrera Atuesta

El sentido práctico de los métodos desarrollados podrá ser apreciado con mayor claridad si se considera su aplicación sobre la estructura del almanaque agrícola D.15b-16b. Para los efectos, se reproducen los intervalos ilustrados en la Figura 6, partiendo del origen 10.17.5.1.2 $1 \mathrm{I} \mathrm{k}^{\prime} 0$ Yax, abril 24 de 1170 (ver Tabla 4), conforme se ilustra en la Figura 8. Las Tablas 8 y 9 describen en detalle las estructuras obtenidas para cada una de las fechas alcanzadas (abril 11 de 1180, y abril 11 de 1222). Al respecto, debe tenerse presente que la validez efectiva del almanaque D.15b-16b estará dada en términos de la fecha solar abril 24 y la componente Jaab' 0 Yax, en el sentido de que ambas deben seguir perteneciendo al intervalo de $13^{\mathrm{d}}$ comprendido entre los límites rituales (inclusivos) $1 \mathrm{I} \mathrm{k}^{\prime}$ y $1 \mathrm{Men}$ si se desea conservar la concordancia iconográfica. 
La estructura del almanaque D.15b-16b obtenida al avanzar $3640^{\mathrm{d}}$ desde abril 24 de 1170 , inicia en 10.17.15.3.2 $1 \mathrm{Ik}$ ' $10 \mathrm{Ch}$ 'en, y se correlaciona con la fecha solar abril 11 de 1180 (Tabla 8; Figura 8). 10 Ch'en precede por $10^{\mathrm{d}}$ a 0 Yax, y abril 11 precede por $13^{\mathrm{d}}$ a abril 24. Por consiguiente, 10.17.15.3.12 11 Eb 0 Yax, y 10.17.15.3.15 1 Men 3 Yax (abril 24 de 1180), siguen perteneciendo al intervalo de $13 \mathrm{~d}$ delimitado por las fechas rituales $1 \mathrm{I}$ ' y $1 \mathrm{Men}$, y como consecuencia de ello la estructura del almanaque agrícola D.15b-16b continúa siendo válida.

La proyección de $18980^{\mathrm{d}}$ a partir de 10.17.5.1.2 $1 \mathrm{I} \mathrm{k}^{\prime} 0$ Yax conduce, por su parte, hacia una estructura con origen en 10.19.17.14.2 1 Ik' 0 Yax (Tabla 9; Figura 8), en la que abril 24 de 1222 se sitúa sobre el extremo opuesto 1 Men del intervalo de $13^{\mathrm{d}}$, indicando que se ha alcanzado la vigencia máxima del almanaque.

\begin{tabular}{|c|c|c|c|c|c|c|c|}
\hline \multicolumn{2}{|l|}{ A } & \multicolumn{2}{|l|}{ B } & \multicolumn{2}{|l|}{ C } & \multicolumn{2}{|l|}{ D } \\
\hline $\begin{array}{l}1 \text { lk' } \\
\text { (10 Ch'en) } \\
10.17 .15 .3 .2 \\
\text { Abr. } 111180\end{array}$ & +13 & $\begin{array}{l}1 \text { Men } \\
\text { (3 Yax) } \\
10.17 .15 .3 .15 \\
\text { Abr. } 241180\end{array}$ & +31 & $\begin{array}{l}6 \text { Kimi } \\
\text { (14 Sak) } \\
10.17 .15 .5 .6 \\
\text { May. } 251180\end{array}$ & +8 & $\begin{array}{l}1 \text { Ix } \\
(2 \mathrm{Kej}) \\
10.17 .15 .5 .14 \\
\text { Jun. } 21180\end{array}$ & +13 \\
\hline $\begin{array}{l}\text { 1 Manik' } \\
\text { (15 Kej) } \\
10.17 .15 .6 .7 \\
\text { Jun. } 151180\end{array}$ & +13 & $\begin{array}{l}\text { 1 Ajaw } \\
\text { (8 Mak) } \\
10.17 .15 .7 .0 \\
\text { Jun. } 281180\end{array}$ & +31 & $\begin{array}{l}6 \text { Chuwen } \\
\text { (19 K'ank'in) } \\
10.17 .15 .8 .11 \\
\text { Jul. } 291180\end{array}$ & +8 & $\begin{array}{l}\text { 1 Kawak } \\
\text { (7 Muwan) } \\
\text { 10.17.15.8.19 } \\
\text { Ago. } 61180\end{array}$ & +13 \\
\hline $\begin{array}{l}1 \text { Eb' } \\
\text { (0 Pax) } \\
\text { 10.17.15.9.12 } \\
\text { Ago. } 191180\end{array}$ & +13 & $\begin{array}{l}\text { 1 Chikchan } \\
\text { (13 Pax) } \\
10.17 .15 .10 .5 \\
\text { Sep. } 11180\end{array}$ & +31 & $\begin{array}{l}6 \text { Kib' } \\
\text { (4 Kumk'u) } \\
\text { 10.17.15.11.16 } \\
\text { Oct. } 21180\end{array}$ & +8 & $\begin{array}{l}\text { 1 K'an } \\
\text { (12 Kumk'u) } \\
10.17 .15 .12 .4 \\
\text { Oct. } 101180\end{array}$ & +13 \\
\hline $\begin{array}{l}\text { 1 Kab'an } \\
\text { (0 Pop) } \\
\text { 10.17.15.12.17 } \\
\text { Oct. } 231180\end{array}$ & +13 & $\begin{array}{l}1 \text { Ok } \\
\text { (13 Pop) } \\
10.17 .15 .13 .10 \\
\text { Nov. } 51180\end{array}$ & +31 & $\begin{array}{l}6 \text { Imix } \\
\text { (4 Sip) } \\
10.17 .15 .15 .1 \\
\text { Dic. } 61180\end{array}$ & +8 & $\begin{array}{l}1 \text { Muluk } \\
\text { (12 Sip) } \\
10.17 .15 .15 .9 \\
\text { Dic. } 141180\end{array}$ & +13 \\
\hline
\end{tabular}

Tabla 8. Fechas de enlace del año 1180 en columnas A y B del almanaque D.15b-16b. (Calendario gregoriano proléptico, constante de correlación 584285; cf. Figuras 4a y 4c.) Autor: Carlos Barrera Atuesta

Arqueológicamente hablando, los registros 10.17.5.1.2 1 Ik' 0 Yax (abril 24 de 1170) y 10.19.17.14.15 1 Men 13 Yax (abril 24 de 1222) establecen el terminus post quem y el terminus ante quem para la operación práctica del almanaque agrícola D.15b-16b, en lo que concierne a su primer elemento iconográfico.

El efecto más evidente del cumplimiento de una Rueda Calendárica es, por consiguiente, la respectiva migración de $13 \mathrm{~d}$ hacia las columnas B y A de la estructura desarrollada para el año 1222 (Tabla 9), de las fechas de enlace que originalmente pertenecían a las columnas A y D del año 1170 (Tabla 4). Este mismo efecto se observa, por su parte, entre las estructuras de los años 1170 y 1180 (cf. Tablas 4 y 8; Figura 8), en virtud de la capacidad predictiva del intervalo de $2 \times 1820^{d}=3640^{d}$. Gemelli Careri (1927) aseveraba que los indios de la Nueva 
España mantenían la correspondencia con el año trópico en el siglo xvII adicionando $13^{\mathrm{d}}$ al fin de 'siglo' de 52 años (entendido como una Rueda Calendárica) para que el primer día del nuevo 'siglo' diera inicio siempre un 10 de abril (cf. Tablas 4 y 9; Figura 8).

\begin{tabular}{|c|c|c|c|c|c|c|c|}
\hline \multicolumn{2}{|l|}{ A } & \multicolumn{2}{|l|}{ B } & \multicolumn{2}{|l|}{ C } & \multicolumn{2}{|l|}{ D } \\
\hline $\begin{array}{l}1 \text { lk' } \\
\text { (0 Yax) } \\
10.19 .17 .14 .2 \\
\text { Abr. } 111222\end{array}$ & +13 & $\begin{array}{l}1 \text { Men } \\
(13 \text { Yax }) \\
10.19 .17 .14 .15 \\
\text { Abr. } 241222\end{array}$ & +31 & $\begin{array}{l}6 \text { Kimi } \\
(4 \mathrm{Kej}) \\
10.19 .17 .16 .6 \\
\text { May. } 251222\end{array}$ & +8 & $\begin{array}{l}1 \mathrm{Ix} \\
(12 \mathrm{Kej}) \\
10.19 .17 .16 .14 \\
\text { Jun. } 21222\end{array}$ & +13 \\
\hline $\begin{array}{l}\text { 1 Manik' } \\
\text { (5 Mak) } \\
\text { 10.19.17.17.7 } \\
\text { Jun. } 151222\end{array}$ & +13 & $\begin{array}{l}1 \text { Ajaw } \\
\text { (18 Mak) } \\
10.19 .18 .0 .0 \\
\text { Jun. } 281222\end{array}$ & +31 & $\begin{array}{l}6 \text { Chuwen } \\
\text { (9 Muwan) } \\
\text { 10.19.18.1.11 } \\
\text { Jul. } 291222\end{array}$ & +8 & $\begin{array}{l}\text { 1 Kawak } \\
\text { (17 Muwan) } \\
\text { 10.19.18.1.19 } \\
\text { Ago. } 61222\end{array}$ & +13 \\
\hline $\begin{array}{l}1 \text { Eb' } \\
\text { (10 Pax) } \\
\text { 10.19.18.2.12 } \\
\text { Ago. } 191222\end{array}$ & +13 & $\begin{array}{l}\text { 1 Chikchan } \\
\text { (3 K'ayab') } \\
10.19 .18 .3 .5 \\
\text { Sep. } 11222\end{array}$ & +31 & $\begin{array}{l}6 \text { Kib' } \\
\text { (14 Kumk'u) } \\
10.19 .18 .4 .16 \\
\text { Oct. } 21222\end{array}$ & +8 & $\begin{array}{l}1 \text { K'an } \\
\text { (2 Wayeb') } \\
10.19 .18 .5 .4 \\
\text { Oct. } 101222\end{array}$ & +13 \\
\hline $\begin{array}{l}\text { 1 Kab'an } \\
\text { (10 Pop) } \\
10.19 .18 .5 .17 \\
\text { Oct. } 231222\end{array}$ & +13 & $\begin{array}{l}1 \text { Ok } \\
\text { (3 Wo) } \\
10.19 .18 .6 .10 \\
\text { Nov. } 51222\end{array}$ & +31 & $\begin{array}{l}6 \text { Imix } \\
\text { (14 Sip) } \\
10.19 .18 .8 .1 \\
\text { Dic. } 61222\end{array}$ & +8 & $\begin{array}{l}1 \text { Muluk } \\
(2 \text { Sotz') } \\
10.19 .18 .8 .9 \\
\text { Dic. } 141222\end{array}$ & +13 \\
\hline
\end{tabular}

Tabla 9. Fechas de enlace del año 1222 en columnas A y B del almanaque D.15b-16b. (Calendario gregoriano proléptico, constante de correlación 584285; cf. Figuras 4a y 4c.) Autor: Carlos Barrera Atuesta

\section{Concordancia etnohistórica}

En su obra Relación de las cosas de Yucatán, Diego de Landa (1864) establece la correspondencia entre el primer día del año maya, y julio 26 de 1553 del calendario gregoriano proléptico (julio 16 del calendario juliano), hecho que permite fijar la culminación del 'mes' Mak y sus respectivos rituales agrarios de fuego y agua ( $c f$. Morales, 2014) para abril 11. Esta fecha se encuentra en concordancia con el primer registro solar obtenido para el almanaque agrícola D.15b-16b, luego de aplicar sobre su estructura del año 1170 (Tabla 4) sendos intervalos de $3640^{\mathrm{d}} \mathrm{y}$ 18980d (Tablas 8 y 9; Figura 8). Abril 11 también pertenece al Grupo 4 de orientaciones de Šprajc que suelen enfilar hacia el oeste (Sánchez y Šprajc, 2015: 74, Tabla 7).

Conforme se argumenta en la sección titulada "módulo aritmético", la culminación en abril 11 del mes yucateco Mak — caracterizado por los procesos agrícolas de roza, tumba y quema- debió coincidir con el origen del período siguiente, dedicado al acopio de semillas, la remoción y siembra del terreno, actividades que se habrían prolongado durante dos trecenas más de días (cf. Tablas 4 , 
8 y 9), finalizando en mayo 7 , poco después del inicio de la temporada de lluvias. Mayo 7, por su parte, pertenece al Grupo 9 de orientaciones astronómicas de Šprajc que suelen enfilar hacia el oeste (Sánchez y Šprajc, 2015: 74, Tabla 7). La iconografía asociada al siguiente período de $31^{\mathrm{d}}$ en la estructura del calendario D.15b-16b (ver Figura 5; Tabla 4) parece simbolizar en la figura de Jun Ajaw el tiempo de transformación durante el cual la semilla "muere" para brotar o "renacer" como planta.

Obsérvese asimismo la inscripción cronológica 9.19.15.0.0 1 Ajaw 3 Sek en la Estela 8 de Machaquilá (Tabla 7), correlacionada con abril 10 de 825 (cf. Inicio del nuevo 'siglo' según Gemelli, 1927) y precedida de abril 11 de 783 (9.17.12.7.0 1 Ajaw 13 Sotz') por $15340^{\mathrm{d}}$ (Figura 8; Tablas 8 y 9: columna A), así como el registro 9.19.10.0.0 8 Ajaw 8 Xul de mayo 6 de 820 en la Estela 4 de Machaquilá (Tabla 7), precedido de mayo 7 de 778 (9.17.7.7.0 8 Ajaw 18 Sek) por 59 ciclos rituales de $260 d$ ( $c f$. Tabla 4: columna B).

\section{Coherencia cronológica}

En julio de 2012, Christian Prager circuló una breve nota epigráfica donde examinaba la posible datación y procedencia del Códice de Dresde mediante la comparación de registros paleográficos de distintas secciones del manuscrito y un fragmento de vasija hallado en la Estructura R-87 de Mayapán, en los que se nombra al dios D de Schellhas —-Itzamná- utilizando estilos caligráficos casi idénticos. Una inscripción calendárica 10 Ajaw 8 Ajaw proveniente de los fragmentos de una escultura de tortuga hallada en la superficie de la Ofrenda 3 y la datación por radiocarbono de otras muestras halladas en el mismo sitio permitieron establecer un marco cronológico comprendido entre 10.18.0.0.0 (10 Ajaw) y 10.19.0.0.0 (8 Ajaw), que se correlaciona con los años cristianos 1185 al 1204. Por consiguiente, la datación establecida por Prager (2012) concuerda con el contexto cronológico del almanaque D.15b-16b, utilizado para fines agrícolas y rituales entre los años 1170 y 1222 (Tablas 4 y 9).

\section{Proyecciones simétricas}

Los estudios que involucran estructuras orientadas hacia abril 11-12 y agosto 31-septiembre 1 ( $c f$. Tablas 8 y 9: columnas Ay B) se remontan a 1916, año en el que Robert Wheeler Willson escribe a Sylvanus Griswold Morley para informarle que, de acuerdo con sus cómputos sobre Copán y según podía observarse desde la Estela 12, el Sol tendría que ocultarse tras la Estela 10 "20.3 días después del equinoccio de primavera y 20.6 días antes del equinoccio de otoño" (Carter, 2014: 35). 


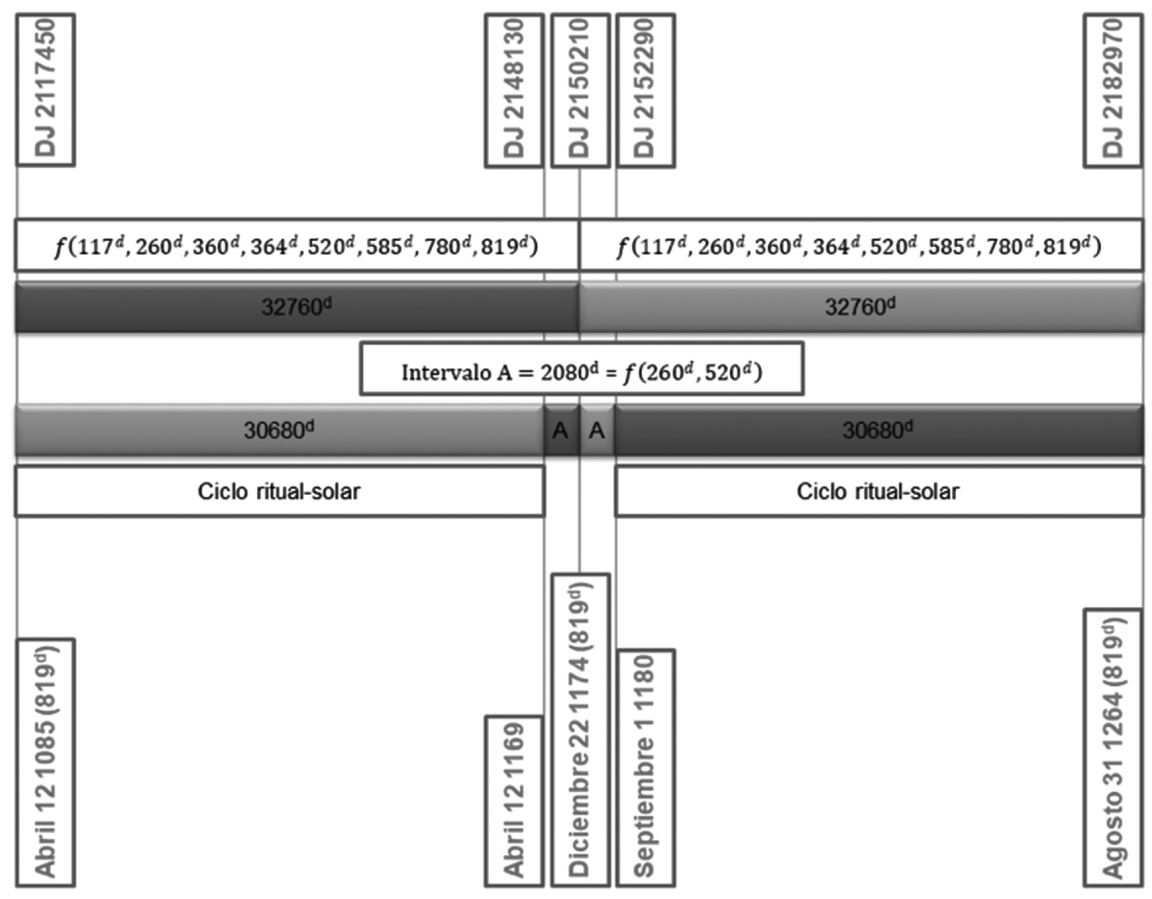

Figura 9. Estructuras simétricas de tiempo vinculadas a septiembre 1 de 1180 a través de intervalos congruentes de $2080^{\mathrm{d}}$ y $32760 \mathrm{~d}$ ( $c f$. Tabla 8: columna B). Autor: Carlos Barrera Atuesta

Diversas investigaciones desde entonces han confirmado la validez general de las orientaciones hacia abril 11 y septiembre 1 , y su relación aproximada con los equinoccios o con los “días de cuarto del año" (marzo 22-23, septiembre 20-21), considerando márgenes de error de $\pm 1^{\mathrm{d}}$ (Sánchez y Šprajc, 2015: Tabla 7; Aldana y Fash, 2014: 34; Martz y Pérez, 2014: Cuadro 2; Green, 2014: Tablas 1.1 y 1.2; Aveni, 2013: 343s; Grofe, 2012: 56s; Aveni y Hartung, 1976; Hohmann y Vogrin, 1982; Tichy, 1978; Drucker, 1977; Hartung, 1968; Morley, 1920).

Los métodos aquí presentados establecen relaciones de simetría respecto a los solsticios, usando intervalos múltiplos de $65 \mathrm{~d}$ y $260 \mathrm{~d}$ y algunos principios de congruencia (ya tratados) que proyectan en el tiempo referencias solares similares. En la Tabla 8, columna B, septiembre 1 de 1180 se encuentra precedido por ocho ciclos rituales de $260^{\mathrm{d}}$ del solsticio de invierno (+1d) del año 1174 (cf. Figura 4a). En virtud de esta pequeña desviación solar, diciembre 22 de 1174 coincide con la efeméride de 819d 10.17.9.14.5 1 Chikchan $18 \mathrm{Sip}$, un hecho que facilita la proyección simétrica de los pares solares abril 11-12 vs. agosto 31-septiembre 1 , por medio de intervalos congruentes de $2080^{\mathrm{d}}$ y $32760^{\mathrm{d}}$ que son función de diversos ciclos mayas, como $117^{\mathrm{d}}, 260^{\mathrm{d}}, 360^{\mathrm{d}}, 520^{\mathrm{d}}, 585^{\mathrm{d}}, 780^{\mathrm{d}}$ y $819^{\mathrm{d}}$ (Figura 9). 


\section{Coordinaciones astronómicas}

Las múltiples correspondencias calendáricas, canónicas y astronómicas descritas por los ciclos congruentes facilitan en gran medida la proyección de fechas y eventos que los mayas pudieron haber considerado como significativos. Valórese el caso del registro 10.19.18.0.0 obtenido para el almanaque agrícola D.15b-16b (Tabla 9: columna B) en donde la componente ritual 1 Ajaw sugiere su asociación con el primer día en que Venus es visible como lucero del alba (Códice de Dresde, 24, 46-50), y la fecha de enlace junio 28 (de 1222) se sitúa 1820d antes de un solsticio de verano ( $c f$. Figura 4a). La transición de 1820d comprendida entre 10.19.18.0.0 1 Ajaw 18 Mak y 11.0.3.1.0 1 Ajaw 13 Mak conduce de facto hacia el mismo registro de junio 22 de 1227 obtenido por Floyd Glenn Lounsbury (1983) para el primer día de visibilidad de Venus como lucero del alba. Podemos intuir, por tanto, que existe una vinculación viable entre el almanaque agrícola D.15b$16 \mathrm{~b}$ y la Tabla de Venus del Códice de Dresde.

Un análisis de la estructura de $65^{\mathrm{d}} \times 260^{\mathrm{d}}$ del almanaque agrícola D.15b-16b revela de hecho que los registros situados $520^{\mathrm{d}}$ antes y $65^{\mathrm{d}}$ después de 10.19.18.0.0 1 Ajaw 18 Mak deberían corresponder con los primeros días de visibilidad de Venus como lucero del alba. El primero de estos registros (10.19.16.10.0 1 Ajaw $3 \mathrm{Xul}$ ) precede por $2340^{\mathrm{d}}$ a 11.0.3.1.0 1 Ajaw 13 Mak, en conformidad con la ecuación diofántica lineal ' $z=37960 x-2340 y$ ' que rige las estructuras de la Tabla de Venus (Lounsbury, 1978). Esta importante ecuación lineal del tiempo es aún utilizada de forma implícita en modernas investigaciones que combinan operaciones de múltiplos anómalos $\left(9100^{\mathrm{d}}, 33280^{\mathrm{d}}, 68900^{\mathrm{d}}\right.$ y $\left.185120^{\mathrm{d}}\right)$ con distintas repeticiones del ciclo de $37960^{\text {d }}$ de la Tabla de Venus (Aldana, 2016; Bricker y Bricker, 2007; 2011: 163s).

El registro 10.19.18.3.5 1 Chikchan 3 K'ayab' (Tabla 9: columna B) coincide, por su parte, con el primer día de visibilidad de Venus de septiembre 1 de 1222 (cf. Figura 4a) y representa la primera de cuatro transiciones de $585^{\mathrm{d}}$ que conducen hacia 11.0.3.1.0 1 Ajaw 13 Mak, desde 10.19.16.10.0 1 Ajaw 3 Xul. La segunda transición de $585^{\mathrm{d}}$, a partir de 10.19.16.10.0 1 Ajaw $3 \mathrm{Xul}$, no solo conduce hacia el primer día de visibilidad de Venus de 10.19.19.14.10 1 Ok 18 Ch'en en abril 8 de 1224, sino que también sitúa al operador de la tabla en una zona de eclipses vinculada —a través de intervalos de $520^{\mathrm{d}}$ — con otras regiones similares.

Teeple (1931: 38) consideraba que la equivalencia aproximada entre $520^{\mathrm{d}}$ y tres períodos de eclipses de $173,31^{\mathrm{d}}$ no era intencional sino accidental. Hoy día, sin embargo, resulta difícil cuestionar la intencionalidad de intervalos mayas de eclipses como el de $7280^{\mathrm{d}}=14 \times 520^{\mathrm{d}}$ (Códice de Dresde, 64) y $4680^{\mathrm{d}}=9 \times 520^{\mathrm{d}}$ (Códice de Dresde, 58b y 59c). En consecuencia, nótese que la primera transición de $520^{\mathrm{d}}$ a partir de 10.19.19.14.10 $10 \mathrm{O} 18$ Ch'en conduce hacia la víspera de un eclipse solar ocurrido en la fecha juliana septiembre 4 de 1225 (11.0.1.4.10 1 Ok 13 K'ayab' + $1^{\mathrm{d}}$ ), que se encuentra situada $7280^{\mathrm{d}}$ antes y $4680^{\mathrm{d}}$ después de sendos eclipses lunares acontecidos en agosto 9 de 1245 y noviembre 10 de 1212 del calendario juliano (11.1.1.8.10 1 Ok 13 Pax y 10.19.8.4.10 1 Ok 13 Wo). 


\section{Conclusiones}

El artículo examina las semejanzas estructurales existentes entre almanaques rituales de 4 x 65 días en códices mayas, las inscripciones calendáricas de algunos monumentos y un conjunto de fechas pertenecientes a modelos de representación e interpretación solar basados en el estudio de orientaciones astronómicas, cámaras cenitales y calendarios observacionales. Elementos iconográficos en almanaques rituales del Códice de Dresde alusivos a fenómenos climáticos periódicos, relacionados con el ciclo de la siembra, y la vinculación de sus registros con fechas canónicas que delimitan las temporadas de lluvia y de sequía han permitido inferir el uso por parte de los antiguos mayas de intervalos múltiplos de $1820^{\mathrm{d}}$ y $3835^{\mathrm{d}}$, como $3640^{\mathrm{d}}, 7670^{\mathrm{d}}, 7280^{\mathrm{d}}$ y $15340^{\mathrm{d}}$, con capacidad suficiente para proyectar a través del tiempo y de forma coordinada las mismas fechas rituales y solares.

Los registros cronológicos del sitio clásico de Machaquilá, Guatemala, exhiben asimismo fechas de dedicación distanciadas por múltiplos de $1800^{\mathrm{d}}, 1820^{\mathrm{d}}$ y $1825^{\mathrm{d}}$, así como otras inscripciones en las que se registran Ruedas Calendáricas y se describen intervalos de $23400^{\mathrm{d}}$, que evidencian el seguimiento simultáneo de los ciclos solares básicos de $360^{\mathrm{d}}, 364^{\mathrm{d}}$ y $365^{\mathrm{d}}$ en sincronía con el ciclo ritual de $260^{\mathrm{d}}$.

En el ámbito metodológico, el artículo contribuye con dos aspectos que ameritan ser considerados: la aplicación de la aritmética modular en el análisis de fenómenos cíclicos y el desarrollo del concepto de congruencia entre intervalos, fundamental para la síntesis de ciclos rituales-solares. Como consecuencia de ello, ha sido posible determinar que el segundo múltiplo de $1820^{\mathrm{d}}$ pronostica con 42 años de anticipación la fecha solar que será obtenida al término de una Rueda Calendárica.

La estructura del almanaque D.15b-16b presenta concordancias importantes con fechas solares características del ciclo agrícola maya — como abril 11 y mayo 7- que coinciden con los Grupos 4 y 9 de orientaciones astronómicas definidos por Sánchez y Šprajc (2015: 74, tabla 7) para la arquitectura de Mesoamérica.

La Relación de las cosas de Yucatán de Diego de Landa permite establecer que la culminación del 'mes' Mak, asociado con el sistema de roza, tumba y quema, y los rituales agrarios mayas del fuego y del agua (Morales, 2014), aconteció precisamente en abril 11 de 1553 y 1554, justo antes del inicio de la siembra y las primeras lluvias, evidenciando la concordancia etnohistórica adicional entre dichas crónicas y las fechas obtenidas a partir de la estructura del almanaque D.15b-16b.

Con base en este y otros análisis practicados, se concluye finalmente que las fechas solares registradas por distintas estructuras arquitectónicas orientadas hacia el cielo o el horizonte pudieron haber sido determinadas teóricamente -incluso con siglos de anticipación- mediante la coordinación de reconocidos intervalos mayas, manteniendo un apropiado nivel de precisión, y sin haber tenido que recurrir a controvertidos 'métodos de ajuste' (como los 'días intercalares' o los 
'años bisiestos'), que hubiesen terminado por afectar la continuidad y la integridad de sus múltiples ciclos calendáricos sincrónicos — períodos cronológicos o dimensiones de cómputo ritual, calendárico, canónico y astronómico que se desarrollan en perfecta correspondencia temporal con otros procesos o causas-.

\section{Bibliografía}

Aldana, Gerardo

2016 "Discovering Discovery: Chich'en Itza, the Dresden Codex Venus Table and the 10th Century Mayan Astronomical Innovation", Journal of Astronomy in Culture, I (1): 57-76, <https://escholarship.org/uc/item/6cr1s6jd > [Consultado el 22 de julio de 2016].

Aldana, Gerardo y William L. Fash

2014 "Art, Astronomy and Statecraft at Late Classic Copán", Mayan Hieroglyphic Astronomy Collaboratory, MGAC Working Paper 20140902: 1-35, <https://sites.google.com/site/glyphastro/papers/wp-files > [Consultado el 23 de octubre de 2015].

Aveni, Anthony F.

2013 Observadores del cielo en el México antiguo, Jorge Ferreiro (trad.), 2ª ed., México: Fondo de Cultura Económica.

Aveni, Anthony F., Anne S. Dowd y Benjamin Vining

2003 "Maya Calendar Reform? Evidence from Orientations of Specialized Architectural Assemblages", Latin American Antiquity, XIV (2): 159-178. DOI: https:// doi.org/10.2307/3557593.

Aveni, Anthony F. y Horst Hartung

1975 "Ancient Maya Architecture and Planning: Possibilities and Limitations for Astronomical Studies", Native American Astronomy, pp. 111-129, Anthony F. Aveni (ed.). Austin, London: University of Texas Press, <http://adsabs.harvard.edu/abs/1977naa..conf..111H> [Consultado el 12 de abril de 2018].

1976 "Investigación preliminar de las orientaciones astronómicas de Copán”, Yaxkin, I (3): 8-13.

1986 "Maya City Planning and the Calendar", Transactions of the American Philosophical Society, LXXVI (7): 1-87.

Aveni, Anthony F., Steven J. Morandi y Polly A. Peterson

1995 "The Maya Number of Time: Intervalic Time Reckoning in the Maya Codices, Part 1", Journal for the History of Astronomy, Archaeoastronomy Supplement, XXVI (20): S1-S28, <https://goo.gl/DyzLNe> [Consultado el 10 de marzo de 2017]. 
Bolles, David

1990 "The Mayan Calendar: The Solar-Agricultural Year, and Correlation Questions", Mexicon, XII: 85-89, <https://goo.gl/33uhrb> [Consultado el 11 de noviembre de 2017].

Bricker, Victoria R.

1982 "The Origin of the Maya Solar Calendar", Current Anthropology, XXIII (1): 101103. DOI: $10.1086 / 202782$.

2007 "Literary Continuities across the Transformation from Maya Hieroglyphic to Alphabetic Writing”, Proceedings of the American Philosophical Society, CLI (1): 27-42, <https://www.jstor.org/stable/4599042> [Consultado el 18 de enero de 2017].

Bricker, Harvey M. y Victoria R. Bricker

1992 "A Method for Cross-Dating Almanacs with Tables in the Dresden Codex", The Sky in Mayan Literature, pp. 43-86, Anthony F. Aveni (ed.). New York, Oxford: Oxford University Press.

2007 "When was the Dresden Codex Venus Table Efficacious?", Skywatching in the Ancient World. New Perspectives in Cultural Astronomy. Studies in Honor of Anthony F. Aveni, pp. 95-119, Clive Ruggles y Gary Urton (eds.). Boulder: University Press of Colorado.

2011 Astronomy in the Maya Codices. Philadelphia: American Philosophical Society.

Broda, Johanna

1971 "Las fiestas aztecas de los dioses de la lluvia”, Revista Española de Antropología Americana, VI: 245-327, <https://goo.gl/DOjxWh> [Consultado el 18 de febrero de 2017].

1983 "Ciclos agrícolas en el culto: un problema de la correlación del calendario mexica", Calendars in Mesoamerica and Peru: Native American Computations of Time, pp. 145-165, Anthony F. Aveni y Gordon Brotherston (eds.). Oxford: British Archaeological Reports International Series, CLXXIV.

2016 "Astronomía y paisajes rituales en Mesoamérica y los Andes: un enfoque antropológico, interdisciplinario y comparativo", Primera Escuela Interamericana de Astronomía Cultural, pp. 99-134, Sixto R. Giménez Benítez y Cecilia Gómez (eds.). La Plata: Universidad Nacional de La Plata, Facultad de Ciencias Astronómicas y Geofísicas, Observatorio Astronómico de la Plata, <https://goo. $\mathrm{gl} / 7 \mathrm{UlvHh}>$.

Carter, Nicholas P.

2014 "Space, Time and Texts: A Landscape Approach to the Classic Maya Hieroglyphic Record", Archaeologies of Text. Archaeology, Technology, and Ethics, pp. 31-58, Matthew T. Rutz y Morag M. Kersel (eds.). Oxford, Philadelphia: Oxbow Books.

Códice de Dresde

s.f. Manuscrito, Sächsische Landesbibliothek -Staats- und Universitätsbibliothek Dresden (Biblioteca del estado de Dresde - Biblioteca de la Universidad de Dresde), <https://goo.gl/yJxv3c $>$. 
Códice de Madrid

s.f. Manuscrito, Museo de América, Madrid (España), <https://goo.gl/X6hqRf>.

Drucker, R. David

1977 "A Solar Orientation Framework for Teotihuacan", Los procesos de cambio: XV Mesa Redonda, II, pp. 277-284, Anthony F. Aveni (ed.). México: Sociedad Mexicana de Antropología.

Espinosa Villatoro, Miguel

2002 "Nuevas observaciones astronómicas en Edzná, Campeche, México 2001", Los Investigadores de la Cultura Maya, X (1): 54-69, <https://goo.gl/Z1XBiV> [Consultado el 18 de febrero de 2017].

Galindo Trejo, Jesús

2015 "Alineación de estructuras arquitectónicas en Mesoamérica: una práctica ancestral de significado calendárico-astronómico", Diferentes povos, diferentes céus e saberes nas Américas: contribuições da astronomia cultural para a história da ciência, pp. 65-81, Luis Carlos Borges (org.). Rio de Janeiro: Museu de Astronomia e Ciências Afins, <https://goo.gl/BB9iqw

Gemelli Careri, Giovanni Francesco

1927 [1699-1700] Viaje a la Nueva España. Juan Francisco Gemelli Careri, José María de Agreda y Sánchez (trad.). México: Sociedad de Bibliófilos Mexicanos.

Green, Harold H.

2014 "Cosmic Order at Chocola: Implications of Solar Observations of the Eastern Horizon at Chocola, Suchitepequez, Guatemala", Archaeoastronomy and the Maya, pp. 17-39, Gerardo Aldana y Villalobos, y Edwin L. Barnhart (eds.). Oxford, Havertown: Oxbow Books.

Grofe, Michael J.

2012 "The Copán Baseline: K’atun 9.11.0.0.0 and the Three Hearthstones in Orion”, Archaeoastronomy, XXV: 54-84, <https://bit.ly/2v5VXsj> [Consultado el 28 de diciembre de 2016].

Hartung, Horst

1968 "Consideraciones sobre los trazos de centros ceremoniales mayas. Influencia de los conocimientos astronómicos en el acomodo de las construcciones", Verhandlungen des XXXVIII, International Amerikanisten Kongresses, StuttgardMünchen, IV: 17-26, Stuttgart-München: Kommissionsverlag K. Renner.

Hohmann, Hasso y Annegrete Vogrin

1982 Die Architektur von Copán (Honduras): Vermessung, Plandarstellung, Untersuchung der baulichen Elemente und des räumlichen Konzepts. Graz: Akademische Druck und Verlagsanstalt. 
Iwaniszewski, Stanislaw

2013 "The Length of the Year in Maya Calendar and Astronomy", Proceedings of the 20th Conference of the European Society for Astronomy in Culture, pp. 287303, Ivan Šprajc and Peter Pehani (eds.). Ljubljana: Slovene Anthropological Society, <https://goo.gl/Xmuls3>.

Landa, fray Diego de

1864 Relation des choses de Yucatan de Diego de Landa (Relación de las cosas de Yucatán), Arthus Bertrand (ed.). Paris, Londres: Trübner and Company (Collection de documents dans les langues indigènes, 3).

Lounsbury, Floyd G.

1978 "Maya Numeration, Computation, and Calendrical Astronomy", Dictionary of Scientific Biography. New York: Charles Scribner's Sons, XV (S1): 759-818, $<$ https://goo.gl/FT3JMg $>$.

1983 "The Base of the Venus Table of the Dresden Codex, and Its Significance for the Calendar-Correlation Problem", Calendars in Mesoamerica and Peru: Native American Computations of Time, pp.1-26, Anthony F. Aveni y Gordon Brotherston (eds.). Oxford: British Archaeological Reports International Series, CLXXIV.

Martin, Simon and Nikolai Grube

2000 Chronicle of the Maya Kings and Queens: Deciphering the Dynasties of the Ancient Maya. London: Thames \& Hudson.

Martz de la Vega, Hans y Miguel Pérez Negrete

2014 "Tehuacalco como lugar de la memoria. Arqueoastronomía y paisaje. Región Centro de Guerrero", Cuicuilco. Revista de Ciencias Antropológicas, XXI (61): 303-331, < https://goo.gl/NCd5Zm> [Consultado el 11 de marzo de 2017].

Mathews, Peter

2001 "The Dates of Tonina and Dark Horse in its History”, PARI Journal, II (1): 1-6.

Meeus, Jean y Denis Savoie

1992 "The History of the Tropical Year", Journal of the British Astronomical Association, CII (1): 40-42.

Méndez, Alonso

2012 "Day of the Holy Cross: Its Cultural and Astronomical Significance", Cosmology, Calendars, and Horizon-based Astronomy: Papers in Honor of Anthony F. Aveni, Society for American Archaeology, 77th Annual Meeting, Memphis, April 22, 2012, $<$ https://goo.gl/SjKDxY>.

Montero García, Ismael Arturo

2013 El sello del sol en Chichén Itzá. México: Fundación Cultural Armella Spitalier, $<$ https://goo.gl/YaUesW $>$. 
Morales Damián, Manuel Alberto

2014 "Mak, rituales agrarios mayas del fuego y del agua en la Relación de las cosas de Yucatán”, Ilu, Revista de Ciencias de las Religiones, XIX: 173-191. DOI: http://dx.doi.org/10.5209/rev_ILUR.2014.v19.46616.

Morante López, Rubén B.

2001 “Las cámaras astronómicas subterráneas”. Arqueología Mexicana, VII (47): 4651, <https://goo.gl/Gh6vzt>.

Morley, Sylvanus Griswold

1920 The Inscriptions at Copán, Honduras, Carnegie Publication CCXIX, Washington: Carnegie Institution of Washington.

Peden, Robert D.

2004 [1981] “The Mayan Calendar - Why 260 Days?", documento electrónico HTML en posesión del autor, no disponible en el dominio Web original $<$ http://www.spiderorchid.com/mesoamerica/mesoamerica.htm > [consultado el 7 de octubre de 2009].

Prager, Christian

2012 "Dating the Dresden Codex? A Postclassic Codex Style Painting of God D's Name Hieroglyph ITZAN TZ'IKIN from Mayapan, Yucatan (1184 - 1204 AD)", documento en posesión del autor, <https://goo.gl/ekATkR>.

Rice, Prudence M.

2004 Maya Political Science. Time, Astronomy and the Cosmos. Austin: University of Texas Press.

Sánchez Nava, Pedro Francisco e Ivan Šprajc

2015 Orientaciones astronómicas en la arquitectura maya de las tierras bajas. México: Instituto Nacional de Antropología e Historia.

Siarkiewicz, Elzbieta

1995 El tiempo en el Tonalamatl. Varsovia: Cátedra de Estudios Ibéricos, Universidad de Varsovia (Monografías, 3).

Soruco Sáenz, Enrique

1991 "Una cueva ceremonial en Teotihuacan y sus implicaciones astronómicas religiosas", Arqueoastronomía y etnoastronomía en Mesoamérica, pp. 291-296, Johanna Broda, Stanislaw Iwaniszewski, y Lucrecia Maupomé (eds.). Ciudad de México: Instituto de Investigaciones Históricas, Universidad Nacional Autónoma de México, <https://goo.gl/nrErKf>.

Šprajc, Ivan

2000 "Problema de ajustes del año calendárico mesoamericano al año trópico", Anales de Antropología XXXIV (1): pp. 133-160, <https://goo.gl/4sMwHG>. 
2004 "Perpetuating Celestial Order in an Earthly Environment: Astronomy in Mesoamerica around 1000", The World in the Year 1000, pp. 87-102, James Heitzman and Wolfgang Schenkluhn (eds.). New York, Oxford: University Press of America, <https://goo.gl/la33ec >.

2017 "Astronomy, Architecture, and Landscape in Prehispanic Mesoamerica", Journal of Archaeological Research, 26 (2): 197-251. DOI: https://doi.org/10.1007/ s10814-017-9109-z.

Teeple, John E.

1931 "Maya Astronomy", Contributions to American Archaeology, I (2): 29-116. Washington: Carnegie Institution of Washington (403).

Tichy, Franz

1978 "El calendario solar como principio de ordenación del espacio para poblaciones y lugares sagrados", Simposio de la Fundación Alemana para la Investigación Científica, Comunicaciones, XV: 153-164.

1991 Die geordnete Welt indianischer Völker: ein Beispiel von Raumordnung und Zeitordnung im vorkolumbischen Mexiko (El mundo ordenado de los pueblos nativos americanos: un ejemplo de planificación espacial y orden temporal en el México precolombino), Johanna Broda (Mitwirkende). Stuttgart: Franz Steiner Verlag (Das Mexiko-Projekt der Deutschen Forschungsgemeinschaft, 21).

Vail, Gabrielle y Anthony Aveni

2004 The Madrid Codex. New Approaches to Understanding an Ancient Maya Manuscript. Boulder: University Press of Colorado.

Vail, Gabrielle y Christine Hernández

2013 Re-Creating Primordial Time. Foundation Rituals and Mythology in the Postclassic Maya Codices. Boulder: University Press of Colorado.

Vail, Gabrielle y Matthew G. Looper

2015 "World Renewal Rituals among the Postclassic Yucatec Maya and Contemporary Ch'orti' Maya”, Estudios de Cultura Maya, XLV: 121-140. DOI: http:// dx.doi.org/10.19130/iifl.ecm.2015.45.135.

Wood Cano, David y Ofelia Márquez Huitzil

2016 "El Metztlapohualli y los Nueve Señores de la Noche: cómputo de 252 días", Antilha, V (15): 47-106, <https://goo.gl//8qVFz>. [Consultado el 18 de febrero de 2017].

Zaro, Gregory y Jon C. Lohse

2005 "Agricultural Rhythms and Rituals: Ancient Maya Solar Observation in Hinterland Blue Creek, Northwestern Belize", Latin American Antiquity, XVI (1): 81-98. DOI: https://doi.org/10.2307/30042487. 